Discussion Paper No. 790

\title{
AUCTIONS WHERE INCOMES ARE PRIVATE INFORMATION AND PREFERENCES (NON QUASI-LINEAR) ARE COMMON KNOWLEDGE
}

\author{
Krishnendu Ghosh Dastidar
}

September 2010

The Institute of Social and Economic Research

Osaka University

6-1 Mihogaoka, Ibaraki, Osaka 567-0047, Japan 


\title{
Auctions where incomes are private information and preferences (non quasi-linear) are common knowledge ${ }^{1}$
}

\author{
Krishnendu Ghosh Dastidar \\ Centre for Economic Studies and Planning, \\ School of Social Sciences, \\ Jawaharlal Nehru University, \\ New Delhi 110067, INDIA. \\ email : kgd12@yahoo.com, kgd0302@mail.jnu.ac.in
}

September, 2010

\footnotetext{
${ }^{1}$ I am indebted to Masaki Aoyagi, Hikmet Gunay, Claudio Mezzetti, Anjan Mukherji and Makoto Yano for this paper. Comments from Marcus Berliant, Wen Chern, Junichiro Ishida, Noriaki Matsushima, Eiichi Miyagawa, Yasuyuki Miyahara, Ryo Ogawa, Shigehiro Serizawa and Lex Zhao were very helful. The first draft of the paper was written when I was a 'Visiting Foreign Scholar' at the Institute of Social and Economic Research, Osaka University in 2010. ISER provided me with excellent research facilites and stimulating intellectual ambience and I am grateful for that. An earlier version of this paper under the title "Basic auction theory revisited" was presented in seminars at University of Calcutta, ISER, Kyoto University, Kobe University and at the 10th SAET conference in Singapore. The usual disclaimer applies.
} 


\begin{abstract}
We consider a two good world where an individual $i$ with income $m_{i}$ has utility function $u(x, y)$, where $x \in[0, \infty)$ and $y \in\{0,1\}$. We first derive the valuation (maximum price that he is willing to pay for the object) for good $y$ as a function of his income. Then we consider the following problem. Suppose good $x$ is available in a store at a fixed price 1 . Good $y$ can be obtained in an auction. In such a situation we show that bidding ones own valuation is an equilibrium in a second-price auction. With risk neutral bidders and high enough incomes we derive the symmetric equilibrium in first-price and all-pay auctions and show that revenue equivalence fails to hold. With risk neutrality we also show that under mild restrictions, the revenue maximising reserve price is zero for all the three auctions and the all-pay auction with zero reserve price fetches the highest expected revenue. With low enough incomes, we show that under some restrictions, bidding ones own valuation is a symmetric equilibrium even for first-price and all-pay auctions. Here also, the expected revenue is the highest with all-pay auctions.

Journal of Economic Literature classification number D44
\end{abstract}




\section{Introduction}

In the traditional benchmark model of auctions we have the following scenario. There is one indivisible object up for sale and there are $n$ potential bidders. Each bidder's valuation (maximum price that he is willing to pay for the object), $v_{i}$, is private information. In terms of demand function the valuation, $v_{i}$, can be interpreted in the following way. Let $p$ be the price of the indivisible object. Then, the demand for the indivisible object, $D(p)$ is as follows.

$$
D(p)=\left\{\begin{array}{c}
1 \text { if } p \in\left[0, v_{i}\right] \\
0 \text { if } p \in\left(v_{i}, \infty\right)
\end{array}\right.
$$

Bidder $i$ knows his (her) own valuation but does not know others' valuations. He (she) only knows that $\forall j \neq i, V_{j}$ lies in the interval $[\underline{v}, \bar{v}]$ with a known distribution function ${ }^{1}$. Seller also does not know any bidder's valuation. He only knows that for all $i, V_{i}$ lies in the interval $[\underline{v}, \bar{v}]$ with a known distribution function. In a standard auction the object is sold to the highest bidder. The payment by each bidder depends on the type of auction used by the seller. There is a huge literature around this model ${ }^{2}$. One of the most celebrated results is the revenue equivalence theorem which states that under certain assumptions (private values, independent types, symmetry, risk neutrality and no budget constraint), the expected revenue to the seller is same across a large class of auctions.

It may be noted that the independent private value model is based on quasilinear utility functions with high enough incomes. In such a case, the demand for the indivisible good and consequently, its valuation is independent of income. As a result, private information about valuations tantamounts to private information about utility functions ${ }^{3}$.

In this paper we consider a more general class of utility functions. We consider a two good world $^{4}$ where an individual $i$ has utility function $u(x, y)$, where $x \in[0, \infty)$ and $y \in\{0,1\}$. The individual's income is $m_{i}$. This $m_{i}$ may be thought of as the total amount of resources (or wealth) available to individual $i$. Under standard assumptions we derive valuation, $v\left(m_{i}\right)$, for good $y$ as a function of his income. This links the budget constraint with valuation. We show that bidder's valuation can never exceed his income. We also show that depending on the nature of the utility

\footnotetext{
${ }^{1}$ As per convention, we use capital letters to denote random variables and corresponding small letters to denote the realised values of such variables.

${ }^{2}$ See Krishna (2010) for all the standard results around the benchmark model.

${ }^{3}$ It does not matter whether incomes are private information or not, as long as they are high enough.

${ }^{4}$ An example would be where $x$ is food and $y$ is a piece of painting.
} 
function and the income level, the valuation can be strictly increasing, constant or even strictly decreasing in income.

We look at the auction problem from a different angle. We assume that all individuals have the same utility function but have different incomes. We treat incomes as types. Each individual's income is private information and this implies that valuations (that are functions of income) are also private information. To the best of our knowledge, no paper till date has taken this approach. Then we consider the following problem. Suppose good $x$ is available in a store at a fixed price 1. One unit of the indivisible good $y$ is sold at an auction house. The price of good $y$ and the winner will be determined in the Bayesian-Nash equilibrium of the auction game. Any individual has the option of not participating in the auction. If he does not participate in the auction for good $y$, he spends his entire income on good $x$ and earns utility $u\left(m_{i}, 0\right)$. Consequently, in any equilibrium the expected payoff to the individual will be at least $u\left(m_{i}, 0\right)$. In no circumstances can a bidder with income $m_{i}$ pay more than $m_{i}$ for any good. If a bidder $i$ were to bid more than $m_{i}$ (in the auction for good $y$ ) and default, then a penalty would be imposed ${ }^{5}$. In such a framework we analyse first-price, second-price and all-pay auctions ${ }^{6}$.

In a second price auction we first show that for any given utility function, choosing a bid equal to valuation is a weakly dominant strategy for any bidder $i$ with income $m_{i}$ (proposition 1 ). This shows that the original Vickrey (1961) result is very robust to changes in the benchmark model.

Unlike the second price auction, we do not have a equilibrium existence result for first-price and all-pay auctions for all possible income levels. As such, to analyse such auctions we need to classify incomes into two categories. In our model we will define a critical level of income $k$ where $k$ is such that $m_{i} \leq k \Leftrightarrow u(0,1) \geq u\left(m_{i}, 0\right)$. If all incomes are below $k$ we say that incomes are low enough and if all incomes are above $k$ we say that incomes are high enough.

We first analyse risk neutral bidders with high enough incomes. We derive the symmetric equilibrium in all the three auctions without any reserve price and show that revenue equivalence fails to hold. More specifically, we show that the expected revenue is same with first price and second price auctions. However, all-pay auction fetches strictly higher expected revenue than first price auction (proposition 2). We try to explain this result by comparing the equilibria in our model with the corresponding equilibria in the symmetric benchmark model. Thereafter, we introduce

\footnotetext{
${ }^{5}$ Che and Gale (1998) has a similar approach.

${ }^{6}$ As usual, one can show that first-price auction is outcome equivalent to Dutch auction and second-price auction is outcome equivalent to English auction.
} 
reserve price and show that under mild restrictions, the revenue maximising reserve price is zero (or no reserve price is optimal) for all the three auctions (proposition 4). This stands in contrast to the benchmark model where the revenue maximising reserve price is typically positive and higher than the minimum possible valuation. Moreover, among the three auctions analysed, the expected revenue to the seller is highest in the all-pay auction with zero reserve price. Whether this is an optimal mechanism or not remains an open question.

We next analyse bidders with low enough incomes. With no reserve price and under some restrictions, we show that bidding ones own valuation is a symmetric equilibrium even for firstprice and all-pay auctions (propositions 5-6). The fact that bidding ones' own valuation can be an equilibrium even in all-pay auctions is surprising and counterintuitive. We also show that in this case the expected revenue is the highest with all-pay auctions and lowest with second price auctions (proposition 7). We illustrate our results with specific numerical examples.

\subsection{Related Literature}

It may be noted that we show for all cases $v_{i}=v\left(m_{i}\right) \leq m_{i}$. While this seems obvious given the nature of our problem, it is important in the context of the literature. We draw attention to an interesting and influential paper by Che and Gale (1998) which drops the assumption of no budget constraint. A simplified version of their model is as follows. As before let $V_{i}=$ bidder $i$ s valuation. But now, in addition, each bidder is subject to an absolute budget of $W_{i}$ which can be strictly less than $V_{i}$. This situation may be possible in a more dynamic context in which a bidder is currently financially constrained, but would like to buy if he could borrow. For example, if bidders are firms they may face borrowing constraints. In no circumstances can a bidder with a value-budget pair $\left(v_{i}, w_{i}\right)$ pay more than $w_{i}$. If a bidder $i$ were to bid more than $w_{i}$ and default, then a penalty would be imposed. Each bider's value-budget pair $\left(V_{i}, W_{i}\right)$ is identically and independently distributed on $[0,1] \times[0,1]$. An important result of Che and Gale (1998) is that under certain conditions the expected revenue in the first price auction is higher than the expected revenue in the second price auction. It may be noted that in our set-up valuations are always less than or equal to income and consequently, we avoid analysing the type of issues dealt with by Che and Gale (1998).

Saitoh and Serizawa (2008) and Sakai (2008) analyse second price auctions on general preference domains (that include non-quasilinear preferences) and show such auctions satisfy efficiency and strategy-proofness. We approach the problem differently and consider a case where preferences 
are common knowledge but budgets are private information. Proposition 1, where we show that choosing a bid equal to valuation is a weakly dominant strategy, is similar to the results derived in Saitoh and Serizawa (2008) and Sakai (2008).

In a very recent paper Gunay, Meng and Nagelberg (2010) analyse auctions with asymmetric bidders in the standard benchmark model and provide sufficient conditions under which the optimal reserve price is zero (or no reserve price is optimal). We have a similar result (proposition 4) and we show that our sufficient conditions are weaker (see the discussion after proposition 4).

Plan of the paper In section 2 we provide the model of our exercise and all the relevant notations. Section 3 provides the equilibrium in second-price auctions. In section 4 we analyse risk-neutral bidders with high enough incomes. Section 5 analyses equilibrium when incomes are low enough. Lastly, the appendix gives the proofs of all the results.

\section{The Model}

We consider a two good world where an individual $i$ has utility function $u(x, y)$ where $x \in[0, \infty)$ and $y \in\{0,1\}$. This means that any non-negative amount of good $x$ can be consumed but in case of good $y$ there are only two choices. Either one unit of good $y$ can be consumed or it cannot be consumed. Individual $i$ 's income is $m_{i}$.

We provide the first set of assumptions. These assumptions impose some standard restrictions on the utility function.

Assumption A1. $u(0,0)=0$ and for all $y \in\{0,1\}, u(x, y)$ is continuous in $x$ for all $x \in[0, \infty)$ and partially differentiable w.r.t. $x$ for all $x \in(0, \infty)$.

Assumption A2. $u(x, y)$ is strictly increasing in both $x$ and $y$. That is, $u(x, 1)>u(x, 0)$ for all $x \in[0, \infty)$. And, for all $y \in\{0,1\}, x_{1}>x_{2} \Leftrightarrow u\left(x_{1}, y\right)>u\left(x_{2}, y\right)$.

Assumption A3. $\lim _{x \rightarrow \infty} u(x, 0)>u(0,1)$.

Note that assumptions A1- A3 imply that $u(0,0)=0<u\left(m_{i}, 0\right)<u\left(m_{i}, 1\right)$ for all $m_{i}>0$. The sign of $u\left(m_{i}, 0\right)-u(0,1)$ is not known. Since $u(x, y)$ is continuous and strictly increasing 
in $x$ and since $\lim _{x \rightarrow \infty} u(x, 0)>u(0,1)$ (see assumption A3) there exists a unique $k>0$ s.t. $u(k, 0)=u(0,1)$.

Hence

$$
u\left(m_{i}, 0\right) \leq u(0,1) \text { iff } m_{i} \leq k
$$

Let price of $x, P_{x}=1$ and price of $y$ be $p$. Hence the individual's problem is

$$
\max _{x \in[0, \infty), y \in\{0,1\}} u(x, y) \quad \text { s.t. } x+p y \leq m_{i} .
$$

There are two possible cases. (i) $m_{i} \leq k$ and (ii) $m_{i}>k$.

We will now derive the valuation for good $y$ (the maximum price that the consumer is willing to pay for $y$ ) for each of these two cases.

\subsection{Valuation for good $y$}

$\underline{\text { Case } 1} m_{i} \leq k \Leftrightarrow u(0,1) \geq u\left(m_{i}, 0\right)$.

It may be noted that if $p \leq m_{i}$ and the individual purchases ${ }^{7} y$ he can spend $m_{i}-p$ on $x$ and get $u\left(m_{i}-p, 1\right)$. If he does not purchase $y$ and spends his entire income on $x$ he gets $u\left(m_{i}, 0\right)$. Here note that for all $p \in\left(0, m_{i}\right]$

$$
u\left(m_{i}, 1\right)>u\left(m_{i}-p, 1\right) \geq u(0,1) \geq u\left(m_{i}, 0\right)
$$

Therefore the individual will buy good $y$ (that is choose $y=1$ ) iff $m_{i}-p \geq 0 \Longleftrightarrow p \leq m_{i}$. Hence, for this case the maximum price he is willing to pay for good $y$ (his valuation for $y$ ) is $v_{i}=m_{i}$.

\footnotetext{
${ }^{7}$ If $p>m_{i}$ then the individual cannot purchase $y$ and he has to spend the entire income on good $x$ and get $u\left(m_{i}, 0\right)$ 
$\underline{\text { Case } 2} m_{i}>k \Leftrightarrow u(0,1)<u\left(m_{i}, 0\right)$

From our assumptions it follows that for each $m_{i}$ there exists a unique $c\left(m_{i}\right)$ s.t. $u\left(c\left(m_{i}\right), 1\right)=$ $u\left(m_{i}, 0\right)$. Clearly $c($.$) is strictly increasing in m_{i}$. As before, if $p \leq m_{i}$ and the individual purchases $y$ he can spend $m_{i}-p$ on $x$ and get $u\left(m_{i}-p, 1\right)$. If he does not purchase $y$ and spends his entire income on $x$ he gets $u\left(m_{i}, 0\right)$. Now

$$
\begin{aligned}
u\left(m_{i}-p, 1\right) & \geq u\left(c\left(m_{i}\right), 1\right)=u\left(m_{i}, 0\right) \\
\quad \text { iff } m_{i}-p & \geq c\left(m_{i}\right) .
\end{aligned}
$$

Therefore the individual will buy good $y$ (that is choose $y=1$ ) iff $m_{i}-p \geq c\left(m_{i}\right) \Longleftrightarrow p \leq$ $m_{i}-c\left(m_{i}\right)$. That is, the maximum price he is willing to pay for good $y$ (his valuation for $y$ ) is $v_{i}=m_{i}-c\left(m_{i}\right)$.

Therefore we get that valuations are functions of income.

$$
v\left(m_{i}\right)=\left\{\begin{array}{c}
m_{i} \text { if } m_{i} \leq k \\
m_{i}-c\left(m_{i}\right) \text { if } m_{i}>k
\end{array}----(1)\right.
$$

where $c\left(m_{i}\right)$ is such that $u\left(c\left(m_{i}\right), 1\right)=u\left(m_{i}, 0\right)$.

In terms of demand function we can write that for an individual with income $m_{i}$ the demand for good $y$ is the following.

$$
D\left(p, m_{i}\right)=\left\{\begin{array}{c}
1 \text { if } p \in\left[0, v\left(m_{i}\right)\right] \\
0 \text { if } p \in\left(v\left(m_{i}\right), \infty\right)
\end{array}\right.
$$

Comment Note that when $m_{i} \in[0, k]$ (income is low enough) the valuation $v_{i}=m_{i}$ is strictly increasing in income. When $m_{i} \in(k, \infty)$ (income is high enough) we claim that the valuation, $v_{i}=m_{i}-c\left(m_{i}\right)$, may be increasing, constant or even decreasing in income. We now illustrate our claim with some examples.

Example 1: Suppose utility is quasilinear in $x$. That is, $u(x, y)=x+\hat{u}(y)$, where $\hat{u}(1)>$ $\hat{u}(0)=0$. Here $u(0,1)=\hat{u}(1)$ and $u\left(m_{i}, 0\right)=m_{i}$. 
Note that here $k=\hat{u}(1)$ and valuation is

$$
v\left(m_{i}\right)=\left\{\begin{array}{c}
m_{i} \text { if } m_{i} \leq k=\hat{u}(1) \\
\hat{u}(1) \text { if } m_{i}>k=\hat{u}(1)
\end{array}\right.
$$

Note that here valuation, $v\left(m_{i}\right)$, is constant for all $m_{i}>k$. In standard auction-theory the above utility function is used and the income is assumed to be high enough (i.e. $m_{i}>k$ ). Incomplete information about others' valuation simply means incomplete information about $\hat{u}(1)$. That is, for the standard independent private value model, each bidder knows his valuation $\left(v_{i}=\hat{u}_{i}(1)\right)$ but does not know other bidders' valuations $\left(v_{j}=\hat{u}_{j}(1), j \neq i\right)$. It may also be noted that since in the benchmark model it is assumed that for all $i, m_{i}>k$; it does not matter whether incomes are private information or not (as valuations do not depend on incomes).

$\underline{\text { Example 2. Let } u(x, y)=x(1+y)+y}$

Note that here $k=1$ and for $m_{i} \in(1, \infty), c\left(m_{i}\right)=\frac{m_{i}-1}{2}$. Hence

$$
v\left(m_{i}\right)=\left\{\begin{array}{c}
m_{i} \text { if } m_{i} \leq k=1 \\
\frac{m_{i}+1}{2} \text { if } m_{i}>k=1
\end{array}\right.
$$

Here $v\left(m_{i}\right)$ is strictly increasing in $m_{i}$ for all $m_{i}$.

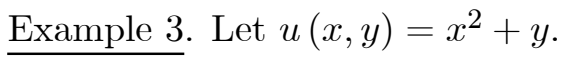

Note that here $k=1$. Hence

$$
v\left(m_{i}\right)=\left\{\begin{array}{c}
m_{i} \text { if } m_{i} \leq k=1 \\
m_{i}-\sqrt{m_{i}^{2}-1} \text { if } m_{i}>k=1
\end{array}\right.
$$

Here $v\left(m_{i}\right)=m_{i}-c\left(m_{i}\right)=m_{i}-\sqrt{m_{i}^{2}-1}$ is strictly decreasing for all $m_{i} \in(1, \infty)$.

\subsection{Introducing incomplete information}

\subsubsection{The basic story}

Our basic story is as follows. Let there be $n$ individuals. All have the same preference ordering (i.e. the same utility function) but different incomes. Bidder $i$ 's income $M_{i}$ is private knowledge to the seller. Bidder $i$ knows that $M_{i}=m_{i}$ but does not know $M_{j}(j \neq i)$. Since valuations are 
function of incomes, private information about incomes tantamounts to private information about valuations. Suppose good $x$ is available at a store at price 1. One unit of good $y$ can be obtained in an auction. We consider three types of auctions viz. first-price, second-price and all-pay auctions. Price of good $y$ will be determined in the Bayesian-Nash equilibrium of the auction. Note that the consumer is no longer a price-taker. His bid affects the equilibrium price of $y$. As noted in the introduction, any individual has the option of not participating in the auction. If he does not participate in the auction for good $y$, he spends his entire income on good $x$ and earns utility $u\left(m_{i}, 0\right)$. Consequently, in any equilibrium, the expected payoff to the individual will be at least $u\left(m_{i}, 0\right)$. In no circumstances can a bidder with income $m_{i}$ pay more than $m_{i}$ for any good. If a bidder $i$ were to bid more than $m_{i}$ (in the auction for good $y$ ) and default, then a penalty, $\gamma$ (where $\gamma>0$ ), would be imposed. If he defaults he cannot obtain good $y$ (even if his bid is the highest). In case the penalty amount exceeds his income, he has to forfeit his income. That is, in case of default, his total payment would be $\min \left\{\gamma, m_{i}\right\}$. If he participates in the auction and makes a payment $P_{i}$ he spends the remaining amount, $m_{i}-P_{i}$, on $\operatorname{good} x$.

We now provide our next set of assumptions.

Assumption B1. Independent types: $M_{1} \ldots . M_{n}$ are independently distributed.

Assumption B2. Symmetry: Each random variable $M_{i} \in[\alpha, \beta]$, where $\alpha \geq 0$, has the same distribution function $F($.$) and associated strictly positive density f($.$) . That is, each bidder i$ believes that competitors' incomes are given by $M_{j} \in[\alpha, \beta]$ with distribution function $F($.$) and$ density function $f($.$) .$

Under assumptions A1-A3 and B1-B2 we will analyse three types of auctions viz (i) first-price auction (ii) second price auction and (iii) all-pay auctions.

Let $r$ be the reserve price. In any such auction the set of actions available for any bidder is $[r, \infty) \cup\{N o\}$ where $\{N o\}$ means that the bidder has the option of not participating in the auction. Individual $i$ 's problem is to choose a strategy $b_{i}\left(m_{i}\right):[\alpha, \beta] \longrightarrow[r, \infty) \cup\{N o\}$ so as to maximise exp. $u(x, y)$. 
remark 1 In first price and all-pay auctions, for any bidder with income $m_{i}$, choosing $\{$ No $\}$ weakly dominates any bid $b_{i}>m_{i}$. For all-pay auctions this is obvious as the bidder has to pay his bid regardless of whether he wins or loses in the auction. For first price auction, the reason is as follows. If the bidder chooses $\{N o\}$ he gets utility $u\left(m_{i}, 0\right)$. If he chooses a bid $b_{i}>m_{i}$ and if his bid is the highest he has to default and pay $\min \left\{\gamma, m_{i}\right\}$. Note that he cannot obtain good $y$ if he defaults. Let $P_{w}$ be the probability of win if he chooses bid $b_{i}>m_{i}$. In this case, his expected payoff is $P_{w} u\left(m_{i}-\min \left\{\gamma, m_{i}\right\}, 0\right)+\left(1-P_{w}\right) u\left(m_{i}, 0\right)$. Note that for any $P_{w} \in[0,1]$ this expected payoff is less than or equal to $u\left(m_{i}, 0\right)$ (with strict inequality when $\left.P_{w}>0\right)$. This implies that it is always better for him to choose $\{N o\}$ than to choose any bid $b_{i}>m_{i}$. This means in first price or all-pay auctions no bidder will choose a bid higher than his income. We will later show that for second price auctions choosing a bid $b_{i}=v\left(m_{i}\right)$ weakly dominates any other bid. Since for all $m_{i} \in[\alpha, \beta]$ we have $v\left(m_{i}\right) \leq m_{i}$, bidders' bids will be below their incomes in second price auctions.

Before giving our main results we need to provide some preliminaries on order statistics.

\subsection{Order Statistics : some notations and preliminaries}

Let $M_{1}, M_{2} . . M_{n}$ denote a random sample of size $n$ drawn from $F($.$) . Then M_{(1)} \geq M_{(2)} \ldots \geq M_{(n)}$ where $M_{(i)} s$ are $M_{i} s$ arranged in decreasing magnitudes, are defined to be the order statistics corresponding to the random sample $M_{1}, M_{2} . . M_{n}$.

We would be interested in $M_{(1)}$ (highest order statistic) and $M_{(2)}$ (second highest order statistic). The corresponding distribution functions and density functions are $F_{1}(),. F_{2}($.$) and f_{1}(),. f_{2}($.$) .$ Note that

$$
\begin{aligned}
F_{1}(z) & =F^{n}(z) \text { and } F_{2}(z)=F^{n}(z)+n\left[F^{n-1}(z)-F^{n}(z)\right] \\
f_{1}(z) & =n F^{n-1}(z) f(z) \text { and } f_{2}(z)=n(n-1) F^{n-2}[1-F(z)] f(z)
\end{aligned}
$$

Another useful random variable is $T=\max \left\{M_{2}, M_{3} \ldots M_{n}\right\}$. To any individual this is the maximum of the others' incomes. The distribution and density function of $T$ is $G($.$) and g($.$) respectively.$ Clearly

$$
\begin{aligned}
G(t) & =F^{n-1}(t) \\
g(t) & =(n-1) F^{n-2}(t) f(t)
\end{aligned}
$$

We will be using these notations frequently in our proofs. 


\section{Equilibrium in second price auctions}

We first analyse second price auction. The result is identical to the standard result in auction theory.

Proposition 1 If $r=0$ then in a second-price auction for any $m_{i} \in[\alpha, \beta]$ it's a weakly dominant strategy for bidder $i$ to bid his own valuation. That is, the following is a symmetric Bayesian Nash equilibrium of the second price auction.

$$
b^{I I}\left(m_{i}\right)=v\left(m_{i}\right)=\left\{\begin{array}{c}
m_{i} \text { if } m_{i} \leq k \\
m_{i}-c\left(m_{i}\right) \text { if } m_{i}>k
\end{array} .\right.
$$

Comment Irrespective of the nature of utility function and the level of incomes, bidding one's own valuation is a weakly dominant strategy in a second price auction. This shows that the original result of Vickrey (1961) on second price auction is very robust to changes in model specifications. As noted in the introduction, Proposition 1 is similar to result derived in Saitoh and Serizawa (2008) and Sakai (2008) that deal with second price auctions on general preference domains (including non-quasilinear preferences) and show such auctions satisfy efficiency and strategy-proofness.

Unlike second price auctions, we do not have a equilibrium existence result for first-price and all-pay auctions for all possible income levels. Consequently, to analyse such auctions we classify incomes into two categories.

High enough and low enough incomes We take $k$ as a critical benchmark of the level of incomes. If $\beta<k$ (that is, all incomes are below $k$ ) then we say that incomes are low enough. If $\alpha \geq k$ (that is, all incomes are above $k$ ) we say that incomes are high enough. 


\section{Risk neutral bidders with high enough incomes}

We now consider the following class of utility functions where the individual is risk neutral and incomes are high enough. In our framework an individual would be risk neutral iff $\frac{\partial^{2}}{\partial x^{2}} u(x, y)=0$. For an explanation about why this is so see Kreps $(1990 \text {, chapter } 3)^{8}$.

It is clear, that, to be consistent with our assumptions, we need a utility function of the following type.

$$
\begin{aligned}
& u(x, y)=\left\{\begin{array}{c}
w x \text { if } y=0 \\
q x+s \text { if } y=1
\end{array}---(2),\right. \\
& \text { where } q \geq w>0 \text { and } s>0 .
\end{aligned}
$$

Note that here $u\left(m_{i}, 0\right)=w m_{i}$ and $u(0,1)=s$. We now explain as to why we need $q \geq w>0$ and $s>0$. First note that if $q<w$ then for large enough $x$ we get $u(x, 0)=w x>q x+s=u(x, 1)$ and this violates assumption A2. If $s=0$ then $u(0,1)=0=u(0,0)$ and this also violates assumption A2.

For this utility function $k=\frac{s}{w}$ and hence from (1) we have

$$
v\left(m_{i}\right)=\left\{\begin{array}{c}
m_{i} \text { if } m_{i} \leq \frac{s}{w} \\
\frac{(q-w) m_{i}+s}{q} \text { if } m_{i}>\frac{s}{w}
\end{array}\right.
$$

Note that if $q=w \quad$ then for all $m_{i}>\frac{s}{w}$ the valuations are constant and $v\left(m_{i}\right)=\frac{s}{w}$ and we are back to the quasi-linear case of standard benchmark model (similar to example 1). Note that in the benchmark model each individual has a different $\frac{s}{w}$ ( say $\left.\frac{s_{i}}{w_{i}}\right)$ and each $\frac{s_{i}}{w_{i}}$ is private information. For this particular case, since the individual $i$ 's valuation $v_{i}\left(m_{i}\right)=\frac{s_{i}}{w_{i}}$ is independent of income (for high enough incomes i.e. for $m_{i}>\frac{s_{i}}{w_{i}}$ ); it does not matter whether incomes are private information or not.

Since we do not consider the quasi-linear utility function we will assume that $q>w$. This means valuations are strictly increasing in incomes for all $m_{i}$. And let $\alpha \geq k=\frac{s}{w}$. This implies that all incomes are high enough.

\footnotetext{
${ }^{8}$ Proposition 3.6 of Kreps (1990) shows that under some standard assumptions the indirect utility function is the von-Neumann-Morgenstern utility functiom for income. Kreps (1990) also shows that the same result goes through if additionally, there is uncertainty over prices. It follows that if the indirect utlity function linear in income then the individual is risk neutral. In our model the indirect utlity function will be linear in income iff $\frac{\partial^{2}}{\partial x^{2}} u(x, y)=0$.
} 


\subsection{Symmetric Bayesian-Nash equilibrium without any reserve price}

We now compute the symmetric Bayesian Nash equilibria for first-price, second-price and all-pay auctions without any reserve price when all incomes are high enough.

\subsubsection{First price auction}

Let $b^{I}\left(m_{i}\right)$, which is strictly increasing in $m_{i}$ be the symmetric Bayesian Nash equilibrium in the first-price auction. Let bidders 2, 3..n choose $b^{I}\left(m_{2}\right), b^{I}\left(m_{3}\right), \ldots b^{I}\left(m_{n}\right)$ and let bidder 1 choose a bid $b_{1}$. Note that $b_{1} \in\left[b^{I}(\alpha), b^{I}(\beta)\right]$. Since $b^{I}($.$) is strictly increasing there exists a z \in[\alpha, \beta]$ such that $b_{1}=b^{I}(z)$. Then the probability that 1 wins is

$$
\begin{aligned}
& \text { Prob. }\left(b_{1}>\max \left\{b^{I}\left(m_{2}\right), b^{I}\left(m_{3}\right), \ldots b^{I}\left(m_{n}\right)\right\}\right) \\
= & \operatorname{Prob} .\left(b^{I}(z)>\max \left\{b^{I}\left(m_{2}\right), b^{I}\left(m_{3}\right), \ldots b^{I}\left(m_{n}\right)\right\}\right) \\
= & \operatorname{Prob} .\left(z>\max \left\{m_{2}, m_{3}, \ldots m_{n}\right\}\right) \\
= & G(z)(\text { see section } 2.3 \text { for the relevant notations })
\end{aligned}
$$

Note that if 1 wins he pays $b^{I}(z)$ to obtain good $y$ and can spend the rest i.e. $m_{1}-b^{I}(z)$ on $\operatorname{good} x$ (whose price is unity). From (2) we get that conditional on winning, his payoff is $q\left(m_{1}-b^{I}(z)\right)+s$. If he does not win the auction he spends his entire income on good $x$ and earns a payoff $w m_{1}$. Hence bidder 1's expected payoff by bidding $b^{I}(z)$ is

$$
\begin{aligned}
\pi_{1}\left(z, m_{1}\right) & =G(z)\left[q\left(m_{1}-b^{I}(z)\right)+s\right]+(1-G(z)) w m_{1} \\
& =G(z)\left[(q-w) m_{1}+s-q b^{I}(z)\right]+w m_{1} .
\end{aligned}
$$

Now for $b^{I}\left(m_{1}\right)$ to be the equilibrium bid chosen by bidder 1 we need that

$$
\begin{aligned}
\frac{\partial \pi_{1}(.)}{\partial z} & =g(z)\left[(q-w) m_{1}+s-q b^{I}(z)\right]-q G(z) \frac{d b^{I}(z)}{d z} \\
& =0 \text { at } z=m_{1} .
\end{aligned}
$$

That is,

$$
g\left(m_{1}\right)\left[(q-w) m_{1}+s-q b^{I}\left(m_{1}\right)\right]-q G\left(m_{1}\right) \frac{d b^{I}\left(m_{1}\right)}{d m_{1}}=0----(3)
$$


Hence $b^{I}\left(m_{1}\right)$ solves the following differential equation (4) and the boundary condition (4a).

$$
\begin{aligned}
\frac{d b^{I}}{d m_{1}} & =\frac{g\left(m_{1}\right)\left[(q-w) m_{1}+s-q b^{I}\right]}{q G\left(m_{1}\right)}---(4) \\
b^{I}(\alpha) & =v(\alpha)=\frac{(q-w) \alpha+s}{q}---(4 a) .
\end{aligned}
$$

To get the exact functional form of $b^{I}(m)$ we proceed as follows. Note that

$$
\begin{aligned}
& \frac{d}{d z}\left[G(z)\left[(q-w) z+s-q b^{I}(z)\right]\right] \\
= & g(z)\left[(q-w) m_{1}+s-q b^{I}(z)\right]+G(z)\left[(q-w)-q \frac{d b^{I}(z)}{d z}\right] \\
= & (q-w) G(z)(\operatorname{using}(3)) .
\end{aligned}
$$

Since $G(\alpha)=0$ we have

$$
\begin{aligned}
& G(z)\left[(q-w) z+s-q b^{I}(z)\right]=\int_{\alpha}^{z}(q-w) G(t) d t \\
& \Rightarrow b^{I}(z)=\frac{1}{q}\left[(q-w) z+s-\frac{(q-w)}{G(z)} \int_{\alpha}^{z} G(t) d t\right] .
\end{aligned}
$$

From section 2.3 we know that $G()=.F^{n-1}($.$) . Hence$

$$
b^{I}(m)=\frac{1}{q}\left[(q-w) m+s-\frac{(q-w)}{F^{n-1}(m)} \int_{\alpha}^{z} F^{n-1}(t) d t\right] .
$$

\subsubsection{Second-price auction}

From proposition 1 we know that when $\alpha \geq k=\frac{s}{q}$ the following constitutes a symmetric Bayesian Nash equilibrium in the second price auction.

$$
b^{I I}(m)=\frac{(q-w) m+s}{q}
$$

\subsubsection{All-Pay auction}

Let $b^{A P}\left(m_{i}\right)$, which is strictly increasing in $m_{i}$ be the symmetric Bayesian Nash equilibrium in the all-pay auction. Let bidders $2,3 . . n$ choose $b^{A P}\left(m_{2}\right), b^{A P}\left(m_{3}\right), \ldots b^{A P}\left(m_{n}\right)$ and as before let bidder 1 choose a bid $b_{1}=b^{A P}(z)$. Consequently, as before the probability that 1 wins is $G(z)$. 
Note that in an all-pay auction bidder 1 pays $b^{A P}(z)$ irrespective of whether he wins or loses. If 1 wins he pays $b^{A P}(z)$ to obtain good $y$ and can spend the rest i.e. $m_{1}-b^{A P}(z)$ on good $x$ (whose price is unity). Conditional on winning, his payoff is $q\left(m_{1}-b^{A P}(z)\right)+s$. If he looses he does not obtain good $y$ but still pays $b^{A P}(z)$ and earns a payoff equal to $w\left(m_{1}-b^{A P}(z)\right)$. Hence, bidder 1 's expected payoff when he bids $b^{A P}(z)$ is

$$
\begin{aligned}
\pi_{1}\left(z, m_{1}\right) & =G(z)\left[q\left(m_{1}-b^{A P}(z)\right)+s\right]+(1-G(z)) w\left(m_{1}-b^{A P}(z)\right) \\
& =G(z)\left[(q-w)\left(m_{1}-b^{A P}(z)\right)+s\right]+w\left(m_{1}-b^{A P}(z)\right) .
\end{aligned}
$$

Now for $b^{A P}\left(m_{1}\right)$ to be the equilibrium bid chosen by bidder 1 we need that

$$
\begin{aligned}
\frac{\partial \pi_{1}(.)}{\partial z} & =g(z)\left[(q-w)\left(m_{1}-b^{A P}(z)\right)+s\right]-(q-w) G(z) \frac{d b^{A P}(z)}{d z}-w \frac{d b^{A P}(z)}{d z} \\
& =0 \text { at } z=m_{1} .
\end{aligned}
$$

That is,

$$
g\left(m_{1}\right)\left[(q-w)\left(m_{1}-b^{A P}\left(m_{1}\right)\right)+s\right]-(q-w) G\left(m_{1}\right) \frac{d b^{A P}\left(m_{1}\right)}{d m_{1}}-w \frac{d b^{A P}\left(m_{1}\right)}{d m_{1}}=0----(5)
$$

Note that in a symmetric equilibrium $b^{A P}(\alpha)=0$. The reason is that any bidder whose income is $\alpha$ wins with probability zero in a symmetric increasing equilibrium. Since it's an all-pay auction he should bid zero and pay zero. Hence $b^{A P}\left(m_{1}\right)$ solves the following differential equation (6) and the boundary condition $(6 a)$.

$$
\begin{aligned}
\frac{d b^{A P}}{d m} & =\frac{g\left(m_{1}\right)\left[(q-w)\left(m_{1}-b^{A P}\right)+s\right]}{G\left(m_{1}\right) q+\left(1-G\left(m_{1}\right)\right) w}---(6) \\
b^{A P}(\alpha) & =0----(6 a) .
\end{aligned}
$$

To get the exact functional form of $b^{A P}(m)$ we proceed as follows. Note that

$$
\begin{aligned}
& \frac{d}{d z}\left[G(z)\left[(q-w)\left(z-b^{A P}(z)\right)+s\right]+w\left(z-b^{A P}(z)\right)\right] \\
= & g(z)\left[(q-w)\left(z-b^{A P}(z)\right)+s\right]+(q-w) G(z)\left(1-\frac{d b^{A P}(z)}{d z}\right)+w\left(1-\frac{d b^{A P}(z)}{d z}\right) \\
= & (q-w) G(z)+w \text { (using } 5)
\end{aligned}
$$

Since $G(\alpha)=0$ and $b^{A P}(\alpha)=0$ we get that

$$
\begin{aligned}
G(z)\left[(q-w)\left(z-b^{A P}(z)\right)+s\right]+w\left(z-b^{A P}(z)\right) & =w \alpha+\int_{\alpha}^{z}[(q-w) G(t)+w] d t \\
14 & =w z+(q-w) \int_{\alpha}^{z} G(t) d t .
\end{aligned}
$$


Noting that $G()=.F^{n-1}($.$) from above we get$

$$
b^{A P}(m)=\frac{F^{n-1}(m)[(q-w) m+s]-(q-w) \int_{\alpha}^{m} F^{n-1}(t) d t}{F^{n-1}(m) q+\left(1-F^{n-1}(m)\right) w} .
$$

remark 2 The derivations of $b^{I}($.$) and b^{A P}$ (.) are only heuristic because both (1) and (3) are necessary conditions. We have not formally established that if the other $(n-1)$ bidders follow $b^{I}($. or $b^{A P}($.$) then it is indeed optimal for a bidder with income m$ to bid $b^{I}(m)$ or $b^{A P}(m)$. It is straightforward to show this and such demonstration is available from the author on request.

\subsubsection{Expected revenues without any reserve price}

The expected revenue in the three auctions (without any reserve price) are as follows.

$$
\begin{aligned}
& \text { First-price }: \quad R^{I}=\int_{\alpha}^{\beta} b^{I}(m) f_{1}(m) d m \\
& \text { Second-price }: \quad R^{I I}=\int_{\alpha}^{\beta} b^{I I}(m) f_{2}(m) d m \\
& \text { All-pay : } \quad R^{A P}=n \int_{\alpha}^{\beta} b^{A P}(m) f(m) d m
\end{aligned}
$$

We now provide our next main result which shows that revenue equivalence breaks down even with risk neutrality.

Proposition 2 When bidders are risk neutral and incomes are high enough (i.e. $\alpha \geq k=\frac{s}{w}$ ) then $R^{A P}>R^{I}=R^{I I}$.

Comment With risk neutral bidders, the expected revenue is same in first-price and second-price auctions for high enough incomes. Surprisingly however, this does not extend to all-pay auctions. This stands in stark contrast to the benchmark model where the expected revenue is same for all three auctions analysed here. We now provide a direct comparison of our result with the benchmark model and provide a plausible explanation behind proposition 2 .

Note that the lowest possible valuation is $v(\alpha)=\frac{(q-w) \alpha+s}{q}$ and the highest possible valuation is $v(\beta)=\frac{(q-w) \beta+s}{q}$. Let $v(\alpha)=\underline{v}$ and $v(\beta)=\bar{v}$. Now consider random variables $v_{i}=v\left(m_{i}\right)$. Since $M_{i}$ s are i.i.d over $[\alpha, \beta]$ with distribution function $F($.$) and density function f($.$) , therefore$ 
$v_{i}$ s are i.i.d over $[\underline{v}, \bar{v}]$ with distribution function $F_{V}(t)=F\left(\frac{q t-s}{q-w}\right)$ and density function $f_{V}(t)=$ $\frac{q-w}{q} f\left(\frac{q t-s}{q-w}\right)$.

Consider any arbitrary bidder with income $m$. Let $v(m)=\rho$ and this implies $m=v^{-1}(\rho)=$ $\frac{q \rho-s}{q-w}$. Now consider a standard auction in the benchmark model where valuations, $v_{i} \mathrm{~s}$, are i.i.d. over $[\underline{v}, \bar{v}]$ with distribution function $F_{V}($.$) and density function f_{V}($.$) .$

In a second price auction of the benchmark model a bidder with valuation $\rho$ would bid $\rho$ and the corresponding bidder in our auction (whose income is $m$ ) would bid $v(m)=\rho$. We now show that the bidding behaviour in first price auction is exactly the same in our model and the benchmark model.

Let in equilibrium a bidder with valuation $\rho$ bid $B^{I}(\rho)$ in the benchmark model. We know that $^{9}$

$$
B^{I}(\rho)=\rho-\int_{\underline{v}}^{\rho} \frac{F_{V}^{n-1}(t)}{F_{V}^{n-1}(\rho)} d t .
$$

Note that $F_{V}^{n-1}(\rho)=F^{n-1}\left(\frac{q \rho-s}{q-w}\right)=F^{n-1}(m)\left(\right.$ since $\left.\rho=v(m)=\frac{(q-w) m+s}{q}\right)$. Also note that $v^{-1}(t)=\frac{q t-s}{q-w}$ and $v^{-1 \prime}(t)=\frac{q}{q-w}$. Hence

$$
\begin{aligned}
\int_{\underline{v}}^{\rho} F_{V}^{n-1}(t) d t & =\left(\frac{q-w}{q}\right)\left(\frac{q}{q-w}\right) \int_{\underline{v}}^{\rho} F^{n-1}\left(\frac{q t-s}{q-w}\right) d t \\
& =\left(\frac{q-w}{q}\right) \int_{\underline{v}}^{\rho} v^{-1 \prime}(t) F^{n-1}\left(v^{-1}(t)\right) d t \\
& =\left(\frac{q-w}{q}\right) \int_{v^{-1}(\underline{v})}^{v^{-1}(\rho)} F^{n-1}(t) d t \\
& =\left(\frac{q-w}{q}\right) \int_{\alpha}^{m} F^{n-1}(t) d t .
\end{aligned}
$$

This implies

$$
\begin{aligned}
B^{I}(\rho) & =v(m)-\left(\frac{q-w}{q}\right)\left(\frac{1}{F^{n-1}(m)}\right) \int_{\alpha}^{m} F^{n-1}(t) d t . \\
& =\frac{(q-w) m+s}{q}-\left(\frac{q-w}{q}\right)\left(\frac{1}{F^{n-1}(m)}\right) \int_{\alpha}^{m} F^{n-1}(t) d t . \\
& =b^{I}(m) .
\end{aligned}
$$

This means that a corresponding bidder whose income is $m$ (where $v(m)=\rho$ ) in our model bids exactly the same as he would have under the benchmark model. Since in the benchmark model the expected revenue in first price auction is the same as in second price auction, the same result must go through in our model also.

\footnotetext{
${ }^{9}$ See chapter 2 of Krishna (2010).
} 
However, now we show that in our model a bidder bids higher in all-pay auction as compared to the corresponding bidder in the benchmark model and this implies that the expected revenue with all-pay auction is higher in our model and revenue equivalence breaks down.

In a symmetric equilibrium in all pay auction under the benchmark model a bidder with valuation $\rho$ bids $B^{A P}(\rho)$ where ${ }^{10}$

$$
B^{A P}(\rho)=F_{V}^{n-1}(\rho) \rho-\int_{\underline{v}}^{\rho} F_{v}^{n-1}(t) d t
$$

From the derivations before we get

$$
\int_{\underline{v}}^{\rho} F_{v}^{n-1}(t) d t=\left(\frac{q-w}{q}\right) \int_{\alpha}^{m} F^{n-1}(t) d t .
$$

We know that $\rho=v(m)$ and this means

$$
F_{V}^{n-1}(\rho)=F^{n-1}\left(\frac{q \rho-s}{q-w}\right)=F^{n-1}(m)
$$

Hence

$$
\begin{aligned}
B^{A P}(\rho) & =F^{n-1}(m) v(m)-\left(\frac{q-w}{q}\right) \int_{\alpha}^{m} F^{n-1}(t) d t \\
& =F^{n-1}(m) \frac{(q-w) m+s}{q}-\left(\frac{q-w}{q}\right) \int_{\alpha}^{m} F^{n-1}(t) d t
\end{aligned}
$$

Note that for all $m \in[\alpha, \beta)$ we have $F^{n-1}(m) q+\left(1-F^{n-1}(m)\right) w<q$. Hence, in our model we have

$$
\begin{aligned}
b^{A P}(m) & =\frac{F^{n-1}(m)[(q-w) m+s]-(q-w) \int_{\alpha}^{m} F^{n-1}(t) d t}{F^{n-1}(m) q+\left(1-F^{n-1}(m)\right) w} \\
& >\frac{F^{n-1}(m)[(q-w) m+s]-(q-w) \int_{\alpha}^{m} F^{n-1}(t) d t}{q} \text { for all } m \in(\alpha, \beta) \\
& =B^{A P}(\rho) .
\end{aligned}
$$

This clearly shows that in an all-pay auction a bidder with income $m$ in our model would bid higher than the corresponding bidder with valuation $\rho$ (where $\rho=v(m)$ ) in the benchmark model. This implies that the expected revenue in all-pay auction would be higher in our model than in the benchmark model. Since in the benchmark model the expected revenue from all-pay auction is the same as in first price or second price auctions, in our model the expected revenue from all-pay auction is higher than either first price or second price auctions.

\footnotetext{
${ }^{10}$ See chapter 3 of Krishna (2010).
} 
We now illustrate proposition 2 with an example.

$\underline{\text { Example } 4}$ Let $u(x, y)=x(1+y)+y$

Here $q=2, w=1$ and $s=1$. Let $\alpha=1, \beta=2$ and let $F($.$) be uniform on [1,2]$. Here $k=1$ and $u\left(m_{i}, 0\right) \geq u(0,1)$ for all $m_{i} \in[1,2]$.

Routine computation shows that $c\left(m_{i}\right)=\frac{m_{i}-1}{2}$ and hence (see $\left.(1)\right)$ we have $v\left(m_{i}\right)=m_{i}-$ $c\left(m_{i}\right)=\frac{m_{i}+1}{2}$. We also have the following.

$$
\begin{aligned}
b^{I}(m) & =\frac{m+3}{4}, b^{I I}(m)=\frac{m+1}{2} \\
\text { and } b^{A P}(m) & =\frac{1}{2} m-\frac{3}{2 m}+1 .
\end{aligned}
$$

The expected revenues are as follows.

$$
\begin{aligned}
R^{I} & =\frac{7}{6}=1.1667 \\
R^{I I} & =\frac{7}{6}=1.1667 \\
R^{A P} & =\frac{7}{2}-3 \ln 2=1.4206 .
\end{aligned}
$$

Clearly all-pay auction fetches more revenue and the revenue equivalence breaks down even with risk neutral bidders.

\subsection{Introducing reserve prices in the risk neutral case}

Let the auctioneer impose a reserve price $r$. This means that bids below $r$ are not acceptable. As before we take $\alpha \geq k=\frac{s}{w}$ (all incomes are high enough). This implies at for all $m_{i} \in[\alpha, \beta]$, $v\left(m_{i}\right)=\frac{(q-w) m_{i}+s}{q}$. It may be noted that without any reserve price we had $b^{I}(\alpha)=v(\alpha)$, $b^{I I}(\alpha)=v(\alpha)$ and $\beta^{A P}(\alpha)=0$. Consequently, any reserve price, $r$, such that $0<r<v(\alpha)$ will have no impact on bidding equilibrium in first-price and second price auctions. However, such a $r$ will affect the equilibrium in all-pay auctions. Also note that, if $r>v(\beta)$ then in any auction the best action for any bidder is to choose $\{N o\}$ (that is, not participate in the auction for good $y$ and spend the entire income on $\operatorname{good} x)$. Hence, we restrict our attention to $r \in[0, v(\beta)]$. We give below the symmetric Bayesian Nash equilibrium for all the three auctions when the seller imposes a positive reserve price $r$. The computation of such equilibria are straightforward. 


\subsubsection{First-Price auction}

If $r \in[0, v(a))$ then for all $m \in[\alpha, \beta]$

$$
b^{I}(m)=\frac{1}{q}\left[(q-w) m+s-\frac{q-w}{F^{n-1}(m)} \int_{\alpha}^{m} F^{n-1}(t) d t\right] \text { for all } m \in[\alpha, \beta] .
$$

If $r \in[v(\alpha), v(\beta)]$ then

$$
b^{I}(m)=\left\{\begin{array}{c}
\{N o\} \text { if } m \in\left[\alpha, \frac{q r-s}{q-w}\right) \\
\frac{1}{q}\left[(q-w) m+s-\frac{q-w}{F^{n-1}(m)} \int_{\frac{q r-s}{q-w}}^{m} F^{n-1}(t) d t\right] \text { if } m \in\left[\frac{q r-s}{q-w}, \beta\right] .
\end{array}\right.
$$

\subsubsection{Second-Price auction}

If $r \in[0, v(a))$ then for all $m \in[\alpha, \beta]$

$$
b^{I I}(m)=\frac{1}{q}[(q-w) m+s] \text { for all } m \in[\alpha, \beta] .
$$

If $r \in[v(\alpha), v(\beta)]$ then

$$
b^{I I}(m)=\left\{\begin{array}{c}
\{N o\} \text { if } m \in\left[\alpha, \frac{q r-s}{q-w}\right) \\
\frac{(q-w) m+s}{q} \text { if } m \in\left[\frac{q r-s}{q-w}, \beta\right] .
\end{array}\right.
$$

Comment As noted before, any $r \in[0, v(a))$ does not affect the bidding strategy in both first price and second price auctions. If $r \in[v(\alpha), v(\beta)]$ then in both auctions a bidder whose income is $v^{-1}(r)=\frac{q r-s}{q-w}$ will be indifferent between bidding and not bidding.

\subsubsection{All-pay auction}

Let $r \in[0, \infty)$ and let in a symmetric equilibrium $\mu$ be the level of income (which depends on $r$ ) such that a bidder with income $\mu$ is indifferent between bidding and not bidding. If the bidder with income $\mu$ chooses not to bid (that is, he selects $\left\{N_{o}\right\}$ ) then he spends his entire income on good $x$ and gets utility $w \mu$. If he bids, he must bid the lowest admissible amount which is $r$. If he bids $r$ then the probability that he wins in a symmetric equilibrium is $F^{n-1}(\mu)$, the probability that all other incomes are less than $\mu$. His expected payoff is then

$$
F^{n-1}(\mu)[q(\mu-r)+s]+\left(1-F^{n-1}(\mu)\right) w(\mu-r)
$$


The above must be equal to $w \mu$, since he is indifferent between bidding and not bidding. That is, we have

$$
F^{n-1}(\mu)[q(\mu-r)+s]+\left(1-F^{n-1}(\mu)\right) w(\mu-r)=w \mu
$$

This implies

$$
F^{n-1}(\mu)[(q-w)(\mu-r)+s]=w r
$$

We can now easily compute that if $r \in[0, v(\beta)]$ then

$$
b^{A P}(m)=\left\{\begin{array}{c}
\{N o\} \text { if } m \in[\alpha, \mu) \\
\frac{F^{n-1}(m)[(q-w) m+s]-(q-w) \int_{\mu}^{m} F^{n-1}(t) d t}{F^{n-1}(m) q+\left(1-F^{n-1}(m)\right) w} \text { if } m \in[\mu, \beta]
\end{array}\right.
$$

where $\mu$ is s.t. $F^{n-1}(\mu)[(q-w)(\mu-r)+s]=w r$.

\subsubsection{Expected revenues with a reserve price}

We now deduce the expected revenue going to the seller for each of the three auctions with a reserve price $r$. In a first price auction the expected payment of a bidder with income $m \geq \frac{q r-s}{q-w}$ is

$$
\begin{aligned}
P^{I}(m, r) & =(\text { Prob. win }) \times b^{I}(m) \\
& =F^{n-1}(m) \times b^{I}(m) . \\
& =F^{n-1}(m) \frac{(q-w) m+s}{q}-\frac{q-w}{q} \int_{\frac{q r-s}{q-w}}^{m} F^{n-1}(t) d t
\end{aligned}
$$

Similarly in a second price auction the expected payment of a bidder with income $m=\frac{q r-s}{q-w}$ is $r F^{n-1}\left(\frac{q r-s}{q-w}\right)$ and the expected payment of a bidder with income $m>\frac{q r-s}{q-w}$ is

$$
P^{I I}(m, r)=r F^{n-1}\left(\frac{q r-s}{q-w}\right)+\int_{\frac{q r-s}{q-w}}^{m} \frac{(q-w) z+s}{q} d F^{n-1}(z)
$$

In an all-pay auction the expected payment of a bidder with income $m \geq \mu$ is just $b^{A P}(m)$. That is,

$$
P^{A P}(m, r)=\frac{F^{n-1}(m)[(q-w) m+s]-(q-w) \int_{\mu}^{m} F^{n-1}(t) d t}{F^{n-1}(m) q+\left(1-F^{n-1}(m)\right) w} .
$$


The expected revenues are as follows.

First-Price Auction:

$$
R^{I}(r)=n \int_{\frac{q r-s}{q-w}}^{\beta} P^{I}(m, r) f(m) d m
$$

Second-Price Auction:

$$
R^{I I}(r)=n \int_{\frac{q r-s}{q-w}}^{\beta} P^{I I}(m, r) f(m) d m
$$

All-Pay Auction:

$$
R^{A P}(r)=n \int_{\mu}^{\beta} P^{A P}(m, r) f(m) d m
$$

where $\mu$ is s.t. $F^{n-1}(\mu)[(q-w)(\mu-r)+s]=w r$.

We now provide our next set of main results that compare expected revenues.

Proposition $3 R^{I}(r)=R^{I I}(r)$ for all $r \in[0, v(\beta)]$.

Comment Like the case of no reserve price, in both second price and first price auctions, one can show that with reserve price $r$, the bidders' equilibrium bids would be exactly the same in our model as they would have in the benchmark model. Since in the symmetric benchmark model, for any reserve price the expected revenue is same in first price and second price auctions, the same holds true for our model as well. However, for any arbitrary $r$, we cannot compare expected revenue in all-pay auction and first-price auction. We show this in terms of an example.

Example 5 We take the same specification as example 4. That is we have the following.

$u(x, y)=x(1+y)+y$.Here $q=2, w=1$ and $s=1$. Let $\alpha=1, \beta=2$ and $F($.$) is uniform$ on $[1,2]$. Here $k=1$ and and hence $v_{i}=m_{i}-c\left(m_{i}\right)=\frac{m_{i}+1}{2}$. Here $v(\alpha)=1$ and $v(\beta)=1.5$. If $r>1.5$ then all bidders will choose $\left\{N_{o}\right\}$ in any of the three auctions. If $r \in[0,1)$ bidding strategies do not change in either first price or second price auctions.

Routine computation shows the following. If $r \in[1,1.5]$ then

$$
b^{I}(m)=\left\{\begin{array}{c}
\{N o\} \text { if } m \in[1,2 r-1) \\
\frac{1}{2} m+\frac{1}{2(m-1)}\left(-\frac{1}{2} m^{2}+m+2 r^{2}-4 r+\frac{3}{2}\right)+\frac{1}{2} \text { if } m \in[2 r-1,2] .
\end{array}\right.
$$

and 


$$
b^{I I}(m)=\left\{\begin{array}{c}
\{N o\} \text { if } m \in[1,2 r-1) \\
\frac{m+1}{2} \text { if } m \in[2 r-1,2]
\end{array} .\right.
$$

For all pay auction we have the following. If $r \in[0,1.5]$ then $\mu=\frac{1}{2} r+\frac{1}{2} \sqrt{r^{2}+4}$ and

$$
b^{A P}(m)=\left\{\begin{array}{c}
\{N o\} \text { if } m \in\left[1, \frac{1}{2} r+\frac{1}{2} \sqrt{r^{2}+4}\right) \\
\frac{1}{m}\left(\begin{array}{c}
m-\frac{1}{2} r+\frac{1}{4} r \sqrt{r^{2}+4}+(m-1)(m+1) \\
-\frac{1}{2} \sqrt{r^{2}+4}-\frac{1}{2} m^{2}+\frac{1}{4} r^{2}+\frac{1}{2}
\end{array}\right) \text { if } m \in\left[\frac{1}{2} r+\frac{1}{2} \sqrt{r^{2}+4}, 2\right]
\end{array}\right.
$$

The expected revenues in first-price and all-pay auctions are as follows.

$$
\begin{gathered}
R^{I}(r)=\left\{\begin{array}{c}
1.1667 \text { if } r \in[0,1] \\
-\frac{16}{3} r^{3}+14 r^{2}-12 r+\frac{9}{2} \text { if } r \in(1,1.5] \\
0 \text { if } r \in(1.5, \infty) .
\end{array}\right. \\
R^{A R}(r)=\left\{\begin{array}{c}
\int_{\frac{1}{2} r+\frac{1}{2} \sqrt{r^{2}+4}}^{2}\left(m-\frac{1}{m} r-\frac{1}{m} \sqrt{r^{2}+4}+\frac{1}{2 m} r^{2}-\frac{1}{m}+\frac{1}{2 m} r \sqrt{r^{2}+4}+2\right) d m \text { if } r \in[0,1.5] \\
0 \text { if } r \in(1.5, \infty) .
\end{array}\right.
\end{gathered}
$$

We plot $R^{I}(r)$ (bold line) and $R^{A R}(r)$ (dash line) below.

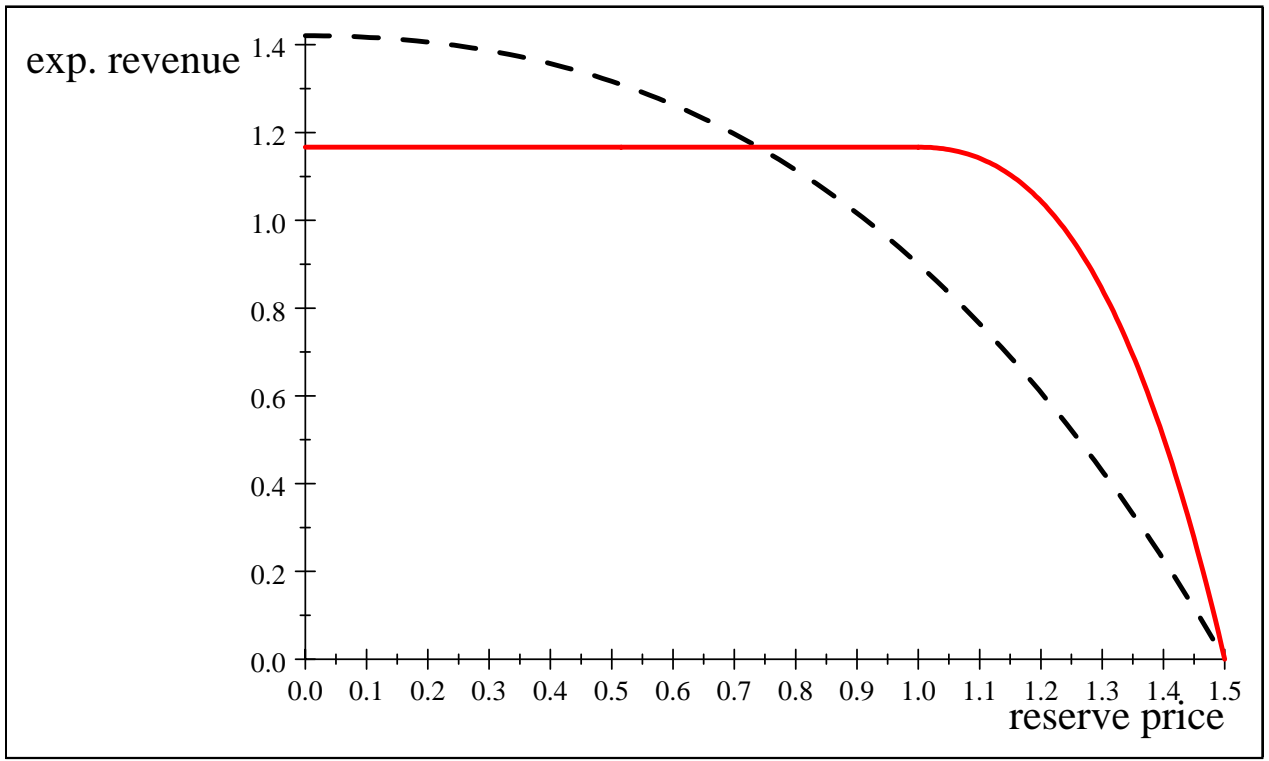

Expected revenue in first price (bold line) and all-pay (dash line) auctions 
The above shows that in our example if $r \in[0.739]$ expected revenue under all-pay auction is higher than under first-price auction and the opposite is true when $r \in[0.739,1.5]$. Hence we cannot have a general revenue ranking for all $r$. It may be noted that in our example, all-pay auctions with zero reserve price fetches the highest revenue among the three auctions analysed. We later show that under some mild restrictions this is always true.

Optimal reserve price We next compute the optimal reserve price in each of these auctions. Let

$$
\begin{aligned}
r^{*}(F P A) & =\arg \max _{r \geq 0} R^{I}(r) \\
r^{*}(S P A) & =\arg \max _{r \geq 0} R^{I I}(r) \\
\text { and } r^{*}(A P A) & =\arg \max _{r \geq 0} R^{A P}(r) .
\end{aligned}
$$

Note that proposition 3 clearly shows that the optimum reserve price will be the same in first-price and second price auction. That is, $r^{*}(F P A)=r^{*}(S P A)$. We now provide our next main result on optimal reserve prices.

Proposition 4 If the hazard rate $\frac{f(.)}{1-F(.)}$ is non-decreasing and if $f(\alpha) \geq \frac{q-w}{(q-w) \alpha+s}$ then

$$
r^{*}(F P A)=r^{*}(S P A)=r^{*}(A P A)=0 .
$$

Comment From proposition 2 we know that $R^{A P}(0)>R^{I}(0)=R^{I I}(0)$. Consequently, from the seller's viewpoint the all pay auction with zero reserve price (or no reserve price) is the best. This stands in contrast to the standard symmetric benchmark model of auctions. Whether or not this is the optimal mechanism in this context remains an open question.

It may also be noted that non-decreasing hazard rate is a very standard assumption in the benchmark auction model. Note that higher is $\alpha$ (the minimum possible income) or higher is $s=u(0,1)$ the lower is $\frac{q-w}{(q-w) \alpha+s}$ and the inequality $f(\alpha) \geq \frac{q-w}{(q-w) \alpha+s}$ is more likely to be satisfied. As such, we can intuitively say that either if all incomes are very high or if $u(0,1)$ is very high then the optimal reserve price will always be zero. 
It may be noted that in the benchmark model the revenue maximising reserve price is generally positive and above $\underline{v}$ (the minimum possible valuation, which is $v(\alpha)$ in our model). As noted in the introduction, recently Gunay, Meng and Nagelberg (2010) analyse reserve prices when bidders are asymmetric. They provide sufficient conditions under which the optimal reserve price is zero. In a symmetric setting their conditions would be the following (see the discussion after proposition 2 in our paper for the notations).

$$
f_{V}^{\prime}(.) \geq 0 \text { and } f_{V}(\underline{v}) \geq \frac{1}{\underline{v}} .
$$

Although our model is different, we show that our sufficient conditions are weaker than Gunay, Meng and Nagelberg (2010). Note that $f_{V}(\underline{v})=\frac{q-w}{q} f\left(\frac{q \underline{v}-s}{q-w}\right)=\frac{q-w}{q} f(\alpha)$ since $\underline{v}=v(\alpha)=$ $\frac{(q-w) \alpha+s}{q}$.Now

$$
\begin{aligned}
f_{V}(\underline{v}) & >\frac{1}{\underline{v}} \\
& \Leftrightarrow \frac{q-w}{q} f(\alpha)>\frac{1}{v(\alpha)} \\
& \Leftrightarrow f(\alpha)>\left(\frac{q}{q-w}\right)\left(\frac{q}{(q-w) \alpha+s}\right) \\
\text { Now RHS } & >\left(\frac{q-w}{q}\right)\left(\frac{q}{(q-w) \alpha+s}\right)=\frac{q-w}{(q-w) \alpha+s} . \\
\text { Hence } f_{V}(\underline{v}) & >\frac{1}{\underline{v}} \Rightarrow f(\alpha)>\frac{q-w}{(q-w) \alpha+s} .
\end{aligned}
$$

Also, $f_{V}^{\prime}(.) \geq 0 \Leftrightarrow f^{\prime}() \geq$.0 and $f^{\prime}() \geq$.0 implies that the hazard rate $\frac{f(.)}{1-F(.)}$ is non-decreasing. Since we need the hazard rate to be non-decreasing and $f(\alpha) \geq \frac{q-w}{(q-w) \alpha+s}$ our sufficient conditions are weaker than the sufficient conditions required in Gunay, Meng and Nagelberg (2010).

\section{Symmetric Equilibria with low enough incomes}

The previous section dealt with risk neutral bidders with high enough incomes. We now turn our attention to the case where bidders' incomes are low enough (i.e. $\beta<k$ ) and there is no reserve price. In this section we allow for general preferences (that include risk neutrality).

Since $\beta<k$ we have $m_{i}<k$ for all $i$ and from (1) we know that $v\left(m_{i}\right)=m_{i}$ for all $i$. We also know from proposition 1 that in a second price auction the symmetric equilibrium will be given by $b^{I I}\left(m_{i}\right)=m_{i}$. We now analyse symmetric equilibria in first-price and all-pay auctions and show that under some restrictions all bidders bidding their valuations is a symmetric Bayesian Nash equilibrium even for these auctions. 
Let us denote the marginal utility of $x$ by the following.

$$
M U_{x}(x, y)=\frac{\partial u(x, y)}{\partial x}=u^{\prime}(x, y) \text { (this is an abuse of notation). }
$$

We now proceed to our next two results.

Proposition 5 If the reverse hazard rate $\frac{f(.)}{F(.)}$ is non-increasing and if for all $x \in[0, \beta]$

$$
u^{\prime}(x, 1) \leq(n-1) f(\beta)[u(0,1)-u(\beta, 0)]
$$

then $b^{I}\left(m_{i}\right)=m_{i}$ is a symmetric Bayesian Nash equilibrium in a first price auction.

Proposition 6 If $\alpha=0$ and if $\forall m_{i} \in[0, \beta]$

$$
\begin{aligned}
F^{n-1}\left(m_{i}\right) u(0,1) & \geq u\left(m_{i}, 0\right) \\
\text { and } F^{n-1}\left(m_{i}\right) u(0,1) & \geq F^{n-1}\left(m_{i}-\varepsilon\right) u(\varepsilon, 1)+\left(1-F^{n-1}\left(m_{i}-\varepsilon\right)\right) u(\varepsilon, 0), \forall \varepsilon \in\left(0, m_{i}\right) .
\end{aligned}
$$

then $b^{A P}\left(m_{i}\right)=m_{i}$ is a symmetric Bayesian Nash equilibrium in an all-pay auction.

The expected revenues in this case are as follows.

$$
\begin{aligned}
\text { First-price }: & R^{I}=\int_{0}^{\beta} z f_{1}(z) d z, \\
\text { Second-price : } & R^{I I}=\int_{0}^{\beta} z f_{2}(z) d z \\
\text { All-Pay : } & R^{A P}=n \int_{0}^{\beta} z f(z) d z
\end{aligned}
$$

We now provide the revenue ranking result for the case when incomes are low enough.

Proposition 7 If all the conditions of propositions 5 and 6 hold then $R^{A P}>R^{I}>R^{I I}$. 
Comment As noted in the introduction, in Che and Gale (1998) an individual's income may be strictly less than his valuation for the auction good. Che and Gale (1998) show that it is possible to have a symmetric equilibrium in a first price auction where bids are equal to incomes and the expected revenue in first price auctions will be strictly higher than the expected revenue in second price auctions. In our model, when incomes are low enough, valuations are exactly equal to incomes (see (1)). We have shown that bidders bid their valuations in a symmetric equilibrium first price auctions (proposition 5). This, together with the fact that $R^{I}>R^{I I}$ (proposition 7 ) is somewhat close in spirit to Che and Gale (1998).

We have also shown that even for all pay auctions it is possible to have a symmetric equilibrium where bidders bid their valuations (proposition 6). This result is surprising and has not been demonstrated before. In a nutshell, when bidders' incomes are low enough, revenue equivalence fails to hold and all-pay auction fetches the highest expected revenue.

We now provide three simple examples to illustrate propositions 5-7. The purpose is twofold.

1. Firstly, the examples show that it is possible to have utility functions and distribution functions that satisfy all conditions of propositions 5 and 6 .

2. Secondly, the examples demonstrate that conditions of propositions 5 and 6 are consistent with (i) risk neutrality (ii) risk aversion and (ii) risk loving.

Example 6a: Bidders are risk neutral. Let $u(x, y)=x+3 y, n=2$ and let $M_{i}$ be uniformly distributed over $[0,1]$. That is, $F(m)=m$. Note that here $k=3 . R^{I}=\frac{2}{3}, R^{I I}=\frac{1}{3}$ and $R^{A P}=1$.

Example 6b: Bidders are risk averse. Let $u(x, y)=\ln (1+x)+3 y, n=2$ and let $M_{i}$ be uniformly distributed over $[0,1]$. That is, $F(m)=m$. Note that here $k=e^{3}-1=19.086 . R^{I}=\frac{2}{3}$, $R^{I I}=\frac{1}{3}$ and $R^{A P}=1$.

Example 6c: Bidders are risk lovers. Let $u(x, y)=x^{2}+3 y, n=2$ and let $M_{i}$ be uniformly distributed over $[0,1]$. That is, $F(m)=m$. Note that here $k=\sqrt{3}=1.7321 . R^{I}=\frac{2}{3}, R^{I I}=\frac{1}{3}$ and $R^{A P}=1$. 


\section{Conclusion}

In this paper we had a re-look at the basic auction model. Unlike the benchmark model we take a novel approach and consider a more general class of utility functions and assume that all individuals have the same utility functions but have different incomes. We treat incomes as types. Each individual's income is private information and this implies that valuations (that are functions of income) are also private information. We have shown that in two good world where one good is available in a store at a fixed price and the other good is sold in an auction, many results of the benchmark model do not hold. While Vickrey's (1961) result on second price auction is very robust, revenue equivalence breaks down even with risk-neutral bidders and high enough incomes.

We propose the following for possible future research.

1. In this paper for first-price and all-pay auctions we have dealt with either high enough incomes (i.e. $\alpha \geq k$ ) or low enough incomes (i.e. $\beta<k$ ). We have not analysed the case when

$\alpha<k<\beta$. Derivation of equilibrium in first price auctions and all-pay auctions, effects of reserve price and revenue ranking results when $\alpha<k<\beta$ should be an interesting (and challenging) future course of research.

2. It would be interesting to compute the expected revenue maximising optimal mechanisms in our model.

3. It would also be interesting to explore the possible equilibria and revenue ranking when bidders are risk averse with high enough incomes.

We believe there is ample scope for further research into this area. 


\section{References}

1. Che, Y-K., and I. Gale (1998) "Standard auctions with financially constrained bidders" Review of Economic Studies, 65, pp. 1-22.

2. Gunay, H., X. Meng and M. Nagelberg (2010) "Reserve prices when bidders are asymmetric" mimeo University of Manitoba.

3. Kreps, D. (1990) "A course in microeconomic theory" Princeton University Press, U.S.A.

4. Krishna, V. (2010) "Auction Theory" (2nd Edition) Academic Press, San Diego, California, U.S.A.

5. Saitoh, H. and S. Serizawa (2008) "Vickrey allocation rule with income effect" Economic Theory 35 pp. 391-401.

6. Sakai, T. (2008) "Second price auctions on general preference domains: two characterisations" Economic Theory 37 pp. 347-356.

7. Vickrey,W. (1961) "Counterspeculation, auctions, and competitive sealed tenders," Journal of Finance, 16, pp. 8-37. 


\section{Appendix}

Proof of Proposition 1 We know that

$$
v\left(m_{i}\right)=\left\{\begin{array}{c}
m_{i} \text { if } m_{i} \leq k \\
m_{i}-c\left(m_{i}\right) \text { if } m_{i}>k
\end{array}\right.
$$

where $c\left(m_{i}\right)$ is such that $u\left(c\left(m_{i}\right), 1\right)=u\left(m_{i}, 0\right)$.

Let's consider bidder 1 whose income is $m_{1}$. Let $z$ be the maximum of the others' bids. Let player 1 bid $b_{1}$. There are two possible cases (i) $m_{1} \leq k$ and (ii) $m_{1}>k$.

Case $1 m_{1} \leq k$. This means $v\left(m_{1}\right)=m_{1}$. For this case there are three possible subcases.

Subcase (i) $z>m_{1}$. If 1 chooses $b_{1}=m_{1}$ he gets a payoff equal to $u\left(m_{1}, 0\right)$ (as he looses in the auction and spends his entire income on good $x$ ). Any $b_{1}<z$ will give him the same payoff. If he bid $b_{1} \geq z$ he wins with a positive probability. Note that if $b_{1}>z$ he wins with certainty and if $b_{1}=z$ there is a tie and his probability of winning is same as the probability of winning the tie. Since $z>m_{1}$ bidder 1 cannot pay for the object after winning it. This means he will default and he has to pay a positive penalty. Hence for this subcase $b_{1}=m_{1}$ is the best.

Subcase (ii) $z<m_{1}$. Choosing a bid $b_{1}=m_{1}$ gives player 1 a payoff equal to $u\left(m_{1}-z, 1\right)$ (as he wins with certainty and pays $z$ for one unit of good $y)$. If he chooses $b_{1} \in\left(z, m_{1}\right)$ he gets the same payoff. Any bid strictly greater than $m_{1}$ will also fetch him the same payoff. If he chooses $b_{1}<z$ he looses the auction and gets $u\left(m_{1}, 0\right)$. Since $m_{1} \leq k$ we have $u\left(m_{1}, 0\right) \leq u(0,1)<u\left(m_{1}-z, 1\right)$. If he chooses $b_{1}=z$ then there is a tie and let $\rho$ be the probability that he wins the tie. His payoff will be $\rho u\left(m_{1}-z, 1\right)+(1-\rho) u\left(m_{1}, 0\right) \leq u\left(m_{1}-z, 1\right)$ for all $\rho \in[0,1]$. Hence his best bid is $b_{1}=m_{1}$.

Subcase (iii) $z=m_{1}$. If he chooses $b_{1}=m_{1}=z$ he gets $\rho u(0,1)+(1-\rho) u\left(m_{1}, 0\right)$. If he chooses $b_{1}<m_{1}=z$ he gets $u\left(m_{1}, 0\right)$. Since $m_{1} \leq k$ we have $u\left(m_{1}, 0\right) \leq u(0,1)$ and hence we have $\rho u(0,1)+(1-\rho) u\left(m_{1}, 0\right) \geq u\left(m_{1}, 0\right)$ for all $\rho \in[0,1]$. If $b_{1}>z=m_{1}$ bidder 1 wins with

certainty but cannot pay for the object after winning it. This means he will default and he has to pay a positive penalty. Hence for this subcase also $b_{1}=m_{1}$ is the best.

Case $2 m_{1}>k$. This means $v\left(m_{1}\right)=m_{1}-c\left(m_{1}\right)$. For this case also there are three possible subcases. 
Subcase (i) $z>v\left(m_{1}\right)$. If $b_{1}=v\left(m_{1}\right)$ bidder 1 looses in the auction and gets $u\left(m_{1}, 0\right)$. Any bid $b_{1}<v\left(m_{1}\right)$ gives him the same payoff. Note that as before we can rule out any bid strictly greater than $m_{1}$. Hence if $z \geq m_{1}$ then bidder 1's best option is to choose a bid $v\left(m_{1}\right)$. If $z<m_{1}$ then if $b_{1} \in\left(z, m_{1}\right]$ will fetch 1 a payoff equal to $u\left(m_{1}-z, 1\right)$. Since $z>v\left(m_{1}\right)$ we have $u\left(m_{1}-z, 1\right)<u\left(m_{1}-v\left(m_{1}\right), 1\right)=u\left(c\left(m_{1}\right), 1\right)=u\left(m_{1}, 0\right)$. Hence $b_{1}=v\left(m_{1}\right)$ is better than $b_{1} \in\left(z, m_{1}\right]$. If $b_{1}=z$ bidder 1 wins with probability $\rho$ and gets

$$
\begin{aligned}
& \rho u\left(m_{1}-z, 1\right)+(1-\rho) u\left(m_{1}, 0\right) \\
\leq & \rho u\left(m_{1}-v\left(m_{1}\right), 1\right)+(1-\rho) u\left(m_{1}, 0\right) \\
= & \rho u\left(c\left(m_{1}\right), 1\right)+(1-\rho) u\left(m_{1}, 0\right) \\
= & u\left(m_{1}, 0\right) \text { since } u\left(c\left(m_{1}\right), 1\right)=u\left(m_{1}, 0\right) .
\end{aligned}
$$

Hence for this subcase $b_{1}=v\left(m_{1}\right)$ is the best.

Subcase (ii) $z<v\left(m_{1}\right)$. Here if $b_{1}=v\left(m_{1}\right)$ bidder 1 wins in the auction and gets $u\left(m_{1}-z, 1\right)$. If he chooses $b_{1} \in\left(z, v\left(m_{1}\right)\right)$ he gets the same payoff. If he chooses $b_{1}<z$ he looses in the auction and gets $u\left(m_{1}, 0\right)$. Note that since $z<v\left(m_{1}\right)$ we have $u\left(m_{1}-z, 1\right)>u\left(m_{1}-v\left(m_{1}\right), 1\right)=$ $u\left(c\left(m_{1}\right), 1\right)=u\left(m_{1}, 0\right)$. Hence $b_{1}=v\left(m_{1}\right)$ is strictly better than $b_{1}<z$. If $b_{1}=z$ bidder 1 wins with probability $\rho$ and gets $\rho u\left(m_{1}-z, 1\right)+(1-\rho) u\left(m_{1}, 0\right) \leq u\left(m_{1}-z, 1\right)$ for all $\rho \in[0,1]$. Hence for this subcase $b_{1}=v\left(m_{1}\right)$ is the best.

Subcase (iii) $z=v\left(m_{1}\right)$. Here if $b_{1}=v\left(m_{1}\right)$ bidder 1 wins in the auction with probability $\rho$ and gets

$$
\begin{aligned}
& \rho u\left(m_{1}-z, 1\right)+(1-\rho) u\left(m_{1}, 0\right) \\
= & \rho u\left(m_{1}-v\left(m_{1}\right), 1\right)+(1-\rho) u\left(m_{1}, 0\right) \\
= & \rho u\left(c\left(m_{1}\right), 1\right)+(1-\rho) u\left(m_{1}, 0\right) \\
= & u\left(m_{1}, 0\right) \text { since } u\left(c\left(m_{1}\right), 1\right)=u\left(m_{1}, 0\right) .
\end{aligned}
$$

If he chooses a bid $b>z=v\left(m_{1}\right)$ then he wins with certainty and gets

$$
u\left(m_{1}-z, 1\right)=u\left(m_{1}-v\left(m_{1}\right), 1\right)=u\left(c\left(m_{1}\right), 1\right)=u\left(m_{1}, 0\right) .
$$

If he chooses $b_{1}<z$ he gets $u\left(m_{1}, 0\right)$. Hence $b_{1}=v\left(m_{1}\right)$ is the best bid for bidder 1 in this subcase. 
Proof of Proposition 2 First note that from section 4.1.1 and 4.1.4 we have

Expected revenue (first price auction) $R^{I}$

$$
\begin{aligned}
& =\int_{\alpha}^{\beta} b^{I}(m) f_{1}(m) d m \\
& =\frac{1}{q} \int_{\alpha}^{\beta}\left[(q-w) m+s-\frac{(q-w)}{F^{n-1}(m)} \int_{\alpha}^{m} F^{n-1}(t) d t\right] f_{1}(m) d m \\
& =\frac{1}{q}\left[\int_{\alpha}^{\beta}[(q-w) m+s] f_{1}(m) d m-(q-w) \int_{\alpha}^{\beta} \frac{1}{F^{n-1}(m)}\left(\int_{\alpha}^{m} F^{n-1}(t) d t\right) f_{1}(m) d m\right]--
\end{aligned}
$$

Also note that

$$
\begin{aligned}
& \int_{\alpha}^{\beta}[(q-w) m+s] f_{1}(m) d m \\
= & (q-w) \int_{\alpha}^{\beta} m f_{1}(m) d m+s----(8) .
\end{aligned}
$$

We also have

$$
\begin{aligned}
& (q-w) \int_{\alpha}^{\beta} \frac{1}{F^{n-1}(m)}\left(\int_{\alpha}^{m} F^{n-1}(t) d t\right) f_{1}(m) d m \\
= & (q-w) \int_{\alpha}^{\beta} \frac{1}{F^{n-1}(m)}\left(\int_{\alpha}^{m} F^{n-1}(t) d t\right) n F^{n-1}(m) f(m) d m \text { (see section 2.3) } \\
= & n(q-w) \int_{\alpha}^{\beta}\left(\int_{\alpha}^{m} F^{n-1}(t) d t\right) f(m) d m \\
= & n(q-w) \int_{\alpha}^{\beta}\left(\int_{t}^{\beta} f(m) d m\right) F^{n-1}(t) d t \text { (by interchanging the order of integration) } \\
= & n(q-w) \int_{\alpha}^{\beta}(1-F(t)) F^{n-1}(t) d t \\
= & (q-w) \int_{\alpha}^{\beta} n(1-F(m)) F^{n-1}(m) d m----(9)
\end{aligned}
$$

Using (8) and (9) in (7) we get

$$
R^{I}=\frac{1}{q}\left[(q-w) \int_{\alpha}^{\beta} m f_{1}(m) d m+s-(q-w) \int_{\alpha}^{\beta} n(1-F(m)) F^{n-1}(m) d m\right]---
$$

This means

$$
R^{I}=\frac{(q-w)}{q}\left[\int_{\alpha}^{\beta} m f_{1}(m) d m-\int_{\alpha}^{\beta} n(1-F(m)) F^{n-1}(m) d m\right]+\frac{s}{q}----(11) .
$$


Note that

$$
\begin{aligned}
R^{I I} & =\int_{\alpha}^{\beta} b^{I I}(m) f_{2}(m) d m \\
& =\int_{\alpha}^{\beta} \frac{(q-w) m+s}{q} f_{2}(m) d m \\
& =\frac{(q-w)}{q} \int_{\alpha}^{\beta} m f_{2}(m) d m+\frac{s}{q}---(12) .
\end{aligned}
$$

Hence

$$
\begin{aligned}
& R^{I}-R^{I I} \\
= & \frac{(q-w)}{q}\left[\int_{\alpha}^{\beta} m f_{1}(m) d m-\int_{\alpha}^{\beta} n(1-F(m)) F^{n-1}(m) d m-\int_{\alpha}^{\beta} m f_{2}(m) d m\right] \\
= & \frac{(q-w)}{q}\left[\int_{\alpha}^{\beta} m d F_{1}(m)-\int_{\alpha}^{\beta} n(1-F(m)) F^{n-1}(m) d m-\int_{\alpha}^{\beta} m d F_{2}(m)\right] \\
= & \frac{(q-w)}{q}\left[\begin{array}{c}
{\left[m F_{1}(m)\right]_{\alpha}^{\beta}-\int_{\alpha}^{\beta} F_{1}(m) d m-\int_{\alpha}^{\beta} n(1-F(m)) F^{n-1}(m) d m} \\
-\left[m F_{2}(m)\right]_{\alpha}^{\beta}+\int_{\alpha}^{\beta} F_{2}(m) d m
\end{array}\right] \\
= & \frac{(q-w)}{q}\left[\begin{array}{c}
\beta-\int_{\alpha}^{\beta} F^{n}(m) d m-\int_{\alpha}^{\beta} n(1-F(m)) F^{n-1}(m) d m \\
-\beta+\int_{\alpha}^{\beta}\left[F^{n}(m)+n F^{n-1}(m)(1-F(m))\right] d m
\end{array}\right] \\
= & 0 .
\end{aligned}
$$

Therefore

$$
R^{I}=R^{I I}
$$

Note that

$$
R^{A P}=n \int_{\alpha}^{\beta} b^{A P}(m) f(m) d m----(13) .
$$

Now from section 4.1.3 we have

$$
b^{A P}(m)=\frac{F^{n-1}(m)[(q-w) m+s]-(q-w) \int_{\alpha}^{m} F^{n-1}(t) d t}{F^{n-1}(m) q+\left(1-F^{n-1}(m)\right) w} .
$$

Note that $q>w$ and $F^{n-1}(m) \in(0,1)$ for all $m \in[\alpha, \beta)$. This means

$$
\text { for all } m \in[\alpha, \beta) \quad q>F^{n-1}(m) q+\left(1-F^{n-1}(m)\right) w
$$

Hence for all $m \in[\alpha, \beta)$

$$
b^{A P}(m)>\frac{1}{q}\left[F^{n-1}(m)[(q-w) m+s]-(q-w) \int_{\alpha}^{m} F^{n-1}(t) d t\right] .
$$


Using above in (13) we get

$$
\begin{aligned}
R^{A P} & >\frac{1}{q} \int_{\alpha}^{\beta}\left[F^{n-1}(m)[(q-w) m+s]-(q-w) \int_{\alpha}^{m} F^{n-1}(t) d t\right] n f(m) d m \\
& =\frac{1}{q}\left[\int_{\alpha}^{\beta}[(q-w) m+s] n F^{n-1}(m) f(m) d m-n(q-w) \int_{\alpha}^{\beta}\left(\int_{\alpha}^{m} F^{n-1}(t) d t\right) f(m) d m\right] \\
& =\frac{1}{q}\left[\int_{\alpha}^{\beta}[(q-w) m+s] f_{1}(m) d m-n(q-w) \int_{\alpha}^{\beta}\left(\int_{\alpha}^{m} F^{n-1}(t) d t\right) f(m) d m\right]--(14) .
\end{aligned}
$$

It may be noted that

$$
\begin{aligned}
& \int_{\alpha}^{\beta}\left(\int_{\alpha}^{m} F^{n-1}(t) d t\right) f(m) d m \\
= & \int_{\alpha}^{\beta}\left(\int_{t}^{\beta} f(m) d m\right) F^{n-1}(t) d t \text { (changing the order of differentiation). } \\
= & \int_{\alpha}^{\beta}(1-F(t)) F^{n-1}(t) d t----(15)
\end{aligned}
$$

Using (15) in (14) we get

$$
\begin{aligned}
R^{A P} & >\frac{1}{q}\left[\int_{\alpha}^{\beta}[(q-w) m+s] f_{1}(m) d m-n(q-w) \int_{\alpha}^{\beta}(1-F(m)) F^{n-1}(m) d m\right] \\
& =R^{I} \text { (using } 8 \text { and 10). }
\end{aligned}
$$

Therefore $R^{A P}>R^{I}=R^{I I}$.

Proof of Proposition 3 When $r \in[0, v(\alpha))$ then it makes no difference to the equilibrium outcome in both first-price auction and second-price auction. That is, $r \in[0, v(\alpha))$ is equivalent to $r=0$. With $r=0$ we know that $R^{I}=R^{I I}$ (from proposition 2). This implies that $R^{I}(r)=R^{I I}(r)$ for all $r \in[0, v(\alpha))$. We now show that the same is true for $r \in[v(\alpha), v(\beta)]$.

From section 4.2.4 we know that for all $m \in\left[\frac{q r-s}{q-w}, \beta\right]$

$$
P^{I}(m, r)=F^{n-1}(m) \frac{(q-w) m+s}{q}-\frac{q-w}{q} \int_{\frac{q r-s}{q-w}}^{m} F^{n-1}(t) d t----(16
$$

Similarly for all $m \in\left[\frac{q r-s}{q-w}, \beta\right]$

$$
P^{I I}(m, r)=r F^{n-1}\left(\frac{q r-s}{q-w}\right)+\int_{\frac{q r-s}{q-w}}^{m} \frac{(q-w) z+s}{q} d F^{n-1}(z)----(16 a)
$$


Now since $F^{n-1}()=.G($.$) we get$

$$
\begin{aligned}
P^{I I}(m, r) & =r G\left(\frac{q r-s}{q-w}\right)+\int_{\frac{q r-s}{q-w}}^{m} \frac{(q-w) z+s}{q} d G(z) \\
& =r G\left(\frac{q r-s}{q-w}\right)+\left[\frac{(q-w) z+s}{q} G(z)\right]_{\frac{q r-s}{q-w}}^{m}-\int_{\frac{q r-s}{q-w}}^{m} G(z) d\left(\frac{(q-w) z+s}{q}\right) \\
& =r G\left(\frac{q r-s}{q-w}\right)+\frac{(q-w) m+s}{q} G(m)-r G\left(\frac{q r-s}{q-w}\right)-\frac{(q-w)}{q} \int_{\frac{q r-s}{q-w}}^{m} G(z) d z \\
& =\frac{(q-w) m+s}{q} G(m)-\frac{(q-w)}{q} \int_{\frac{q r-s}{q-w}}^{m} G(z) d z \\
& =\frac{(q-w) m+s}{q} F^{n-1}(m)-\frac{(q-w)}{q} \int_{\frac{q r-s}{q-w}}^{m} F^{n-1}(z) d z\left(\text { since } F^{n-1}(.)=G(.)\right) \\
& =P^{I}(m, r)(\text { from } 16) .
\end{aligned}
$$

We also know (from section 4.2.4) that for $r \in[v(\alpha), v(\beta)]$

$$
\begin{aligned}
R^{I}(r) & =n \int_{\frac{q r-s}{q-w}}^{\beta} P^{I}(m, r) f(m) d m \\
R^{I I}(r) & =n \int_{\frac{q r-s}{q-w}}^{\beta} P^{I I}(m, r) f(m) d m
\end{aligned}
$$

Since we have just shown that $P^{I}(m, r)=P^{I I}(m, r)$ for all $r \in[v(\alpha), v(\beta)]$, we get that $R^{I}(r)=$ $R^{I I}(r)$ for all $r \in[v(\alpha), v(\beta)]$. At the begining of the proof we have already shown that $R^{I}(r)=$ $R^{I I}(r)$ for all $r \in[0, v(\alpha))$

Proof of Proposition 4 First note that for any $r \in[0, v(\alpha)), R^{I}(r)$ is same as $R^{I}(0)$. And so

$$
r \in[0, v(\alpha)) \text { we have } \frac{d}{d r} R^{I}(r)=0
$$

For $r \in(v(\beta), \infty)$ we have $R^{I}(r)=0$ (since no bidder will make any bid for such a $r$ ).

For $r \in[v(\alpha), v(\beta)]$ we have

$$
\begin{aligned}
\frac{d}{d r} R^{I}(r) & =\frac{d}{d r}\left[n \int_{\frac{q r-s}{q-w}}^{\beta} P^{I}(m, r) f(m) d m\right] \\
& =n\left[-\frac{q}{q-w} P^{I}\left(\frac{q r-s}{q-w}, r\right) f\left(\frac{q r-s}{q-w}\right)+\int_{\frac{q r-s}{q-w}}^{\beta} \frac{\partial}{\partial r}\left(P^{I}(m, r)\right) f(m) d m\right]---(17)
\end{aligned}
$$


Now

$$
\begin{aligned}
P^{I}\left(\frac{q r-s}{q-w}, r\right) & =F^{n-1}\left(\frac{q r-s}{q-w}\right) \frac{(q-w)\left(\frac{q r-s}{q-w}\right)+s}{q} \\
& =r F^{n-1}\left(\frac{q r-s}{q-w}\right)----(18)
\end{aligned}
$$

And

$$
\begin{aligned}
\frac{\partial}{\partial r}\left(P^{I}(m, r)\right) & =\frac{\partial}{\partial r}\left[F^{n-1}(m) \frac{(q-w) m+s}{q}-\frac{q-w}{q} \int_{\frac{q r-s}{q-w}}^{m} F^{n-1}(t) d t\right] \\
& =\frac{q-w}{q} \times \frac{q}{q-w} F^{n-1}\left(\frac{q r-s}{q-w}\right) \\
& =F^{n-1}\left(\frac{q r-s}{q-w}\right)---(19) .
\end{aligned}
$$

Using (18) and (19) in (17) we get that

$$
\begin{aligned}
\frac{d}{d r} R^{I}(r) & =n\left[-\frac{q}{q-w} r F^{n-1}\left(\frac{q r-s}{q-w}\right) f\left(\frac{q r-s}{q-w}\right)+\int_{\frac{q r-s}{q-w}}^{\beta} F^{n-1}\left(\frac{q r-s}{q-w}\right) f(m) d m\right] \\
& =n\left[-\frac{q}{q-w} r F^{n-1}\left(\frac{q r-s}{q-w}\right) f\left(\frac{q r-s}{q-w}\right)+F^{n-1}\left(\frac{q r-s}{q-w}\right) \int_{\frac{q r-s}{q-w}}^{\beta} f(m) d m\right] \\
& =n\left[-\frac{q}{q-w} r F^{n-1}\left(\frac{q r-s}{q-w}\right) f\left(\frac{q r-s}{q-w}\right)+F^{n-1}\left(\frac{q r-s}{q-w}\right)\left(1-F\left(\frac{q r-s}{q-w}\right)\right)\right] \\
& =n F^{n-1}\left(\frac{q r-s}{q-w}\right)\left(1-F\left(\frac{q r-s}{q-w}\right)\right)\left[-\left(\frac{q r}{q-w}\right)\left(\frac{f\left(\frac{q r-s}{q-w}\right)}{1-F\left(\frac{q r-s}{q-w}\right)}\right)+1\right]--(20) .
\end{aligned}
$$

Since $\frac{f(z)}{1-F(z)}$ is non-decreasing in $z$ and $f(\alpha) \geq \frac{q-w}{(q-w) \alpha+s}$ we have

$$
\frac{f\left(\frac{q r-s}{q-w}\right)}{1-F\left(\frac{q r-s}{q-w}\right)} \geq \frac{f(\alpha)}{1-F(\alpha)}=f(\alpha) \geq \frac{q-w}{(q-w) \alpha+s}---(21) .
$$

Now

$$
r \in[v(\alpha), v(\beta)] \Rightarrow r \geq \frac{(q-w) \alpha+s}{q}---(22) .
$$

Using (21) and (22) we get that for $r \in[v(\alpha), v(\beta)]$

$$
\left(\frac{q r}{q-w}\right)\left(\frac{f\left(\frac{q r-s}{q-w}\right)}{1-F\left(\frac{q r-s}{q-w}\right)}\right) \geq\left[\frac{q\left(\frac{(q-w) \alpha+s}{q}\right)}{q-w}\right]\left(\frac{q-w}{(q-w) \alpha+s}\right)=1----(23) .
$$

Using (23) in (20) we get that $\frac{d}{d r} R^{I}(r) \leq 0$ for all $r \in[v(\alpha), v(\beta)]$. Note that $R^{I}(r)=R^{I}(0)$ for all $r \in\left[0, v(\alpha)\right.$ ). This implies that $r^{*}(F P A)=0$. Since $R^{I}(r)=R^{I I}(r)$ for all $r \in[0, v(\beta)]$ (from proposition 3) we also get that $r^{*}(S P A)=0$. 
To compute $r^{*}(A P A)$ note the following. From section 4.2.3 we know that if $r \in[0, v(\beta)]$ then

$$
b^{A P}(m)=\left\{\begin{array}{c}
\{N o\} \text { if } m \in[\alpha, \mu) \\
\frac{F^{n-1}(m)[(q-w) m+s]-(q-w) \int_{\mu}^{m} F^{n-1}(t) d t}{F^{n-1}(m) q+\left(1-F^{n-1}(m)\right) w} \text { if } m \in[\mu, \beta]
\end{array}\right.
$$

where $\mu$ is s.t. $F^{n-1}(\mu)[(q-w(\mu-r)+s]=w r$.

As noted in section 4.2.4, in an all-pay auction the expected payment, $P^{A P}(m, r)$, of a bidder with income $m \geq \mu$ is just $b^{A P}(m)$. That is,

$$
P^{A P}(m, r)=\frac{F^{n-1}(m)[(q-w) m+s]-(q-w) \int_{\mu}^{m} F^{n-1}(t) d t}{F^{n-1}(m) q+\left(1-F^{n-1}(m)\right) w}---(24) .
$$

The expected revenue for any $r \in[0, v(\beta)]$ is as follows.

$$
R^{A P}(r)=n \int_{\mu}^{\beta} P^{A P}(m, r) f(m) d m
$$

Hence

$$
\frac{d}{d r} R^{A P}(r)=n\left[-\frac{d \mu}{d r} P^{A P}(\mu, r) f(\mu)+\int_{\mu}^{\beta} \frac{\partial}{\partial r}\left(P^{A P}(m, r)\right) f(m) d m\right]----
$$

Note that

$$
P^{A P}(\mu, r) f(\mu)=\frac{F^{n-1}(\mu)[(q-w) \mu+s] f(\mu)}{F^{n-1}(\mu) q+\left(1-F^{n-1}(\mu)\right) w}---(26)
$$

Also

$$
\frac{\partial}{\partial r}\left(P^{A P}(m, r)\right)=\frac{(q-w) \frac{d \mu}{d r} F^{n-1}(\mu)}{F^{n-1}(m) q+\left(1-F^{n-1}(m)\right) w}---(27
$$

Note that since since $q>w$ and $\mu \leq m$ we get

$$
F^{n-1}(\mu) q+\left(1-F^{n-1}(\mu)\right) w \leq F^{n-1}(m) q+\left(1-F^{n-1}(m)\right) w .
$$

Using the above in (27) we have

$$
\frac{\partial}{\partial r}\left(P^{A P}(m, r)\right) \leq \frac{(q-w) \frac{d \mu}{d r} F^{n-1}(\mu)}{F^{n-1}(\mu) q+\left(1-F^{n-1}(\mu)\right) w}----
$$


Using (26) and (28) in (25) we get

$$
\begin{aligned}
\frac{d}{d r} R^{A P}(r) & \leq n\left[-\frac{d \mu}{d r} \frac{F^{n-1}(\mu)[(q-w) \mu+s] f(\mu)}{F^{n-1}(\mu) q+\left(1-F^{n-1}(\mu)\right) w}+\frac{\int_{\mu}^{\beta}(q-w) \frac{d \mu}{d r} F^{n-1}(\mu) f(m) d m}{F^{n-1}(\mu) q+\left(1-F^{n-1}(\mu)\right) w}\right] \\
& =\frac{n F^{n-1}(\mu) \frac{d \mu}{d r}}{F^{n-1}(\mu) q+\left(1-F^{n-1}(\mu)\right) w}\left[-[(q-w) \mu+s] f(\mu)+(q-w) \int_{\mu}^{\beta} f(m) d m\right] \\
& =\frac{n F^{n-1}(\mu) \frac{d \mu}{d r}}{F^{n-1}(\mu) q+\left(1-F^{n-1}(\mu)\right) w}[-[(q-w) \mu+s] f(\mu)+(q-w)(1-F(\mu))] \\
& =\frac{n F^{n-1}(\mu) \frac{d \mu}{d r}(1-F(\mu))}{F^{n-1}(\mu) q+\left(1-F^{n-1}(\mu)\right) w}\left[-\frac{[(q-w) \mu+s] f(\mu)}{1-F(\mu)}+(q-w)\right]---(29)
\end{aligned}
$$

From section 4.2 .3 note that since $\mu$ is s.t. $F^{n-1}(\mu)[(q-w(\mu-r)+s]=w r$, we have $\mu \geq \alpha$ and $\frac{d \mu}{d r} \geq 0$. Also, it is given that $\frac{f(z)}{1-F(z)}$ is non-decreasing in $z$ and $f(\alpha) \geq \frac{q-w}{(q-w) \alpha+s}$. So we get

$$
\begin{aligned}
\frac{[(q-w) \mu+s] f(\mu)}{1-F(\mu)} & \geq \frac{[(q-w) \alpha+s] f(\alpha)}{1-F(\alpha)} \\
& =[(q-w) \alpha+s] f(\alpha) \\
& \geq(q-w)---(30)
\end{aligned}
$$

Hence from (29) and (30) we get $\frac{d}{d r} R^{A P}(r) \leq 0$. This implies $r^{*}(A P A)=0$.

Proof of Proposition 5 Let bidders 2, 3...n bid $m_{2}, m_{3} \ldots m_{n}$. Bidder 1's income is $m_{1}$ and he chooses a bid equal to $b_{1}$. Then bidder 1 's expected payoff is

$$
\pi_{1}\left(b_{1}, m_{1}\right)=G\left(b_{1}\right) u\left(m_{1}-b_{1}, 1\right)+\left(1-G\left(b_{1}\right)\right) u\left(m_{1}, 0\right) .
$$

Then

$$
\frac{\partial \pi_{1}(.)}{\partial b_{1}}=g\left(b_{1}\right)\left[u\left(m_{1}-b_{1}, 1\right)-u\left(m_{1}, 0\right)\right]-G\left(b_{1}\right) u^{\prime}\left(m_{1}-b_{1}, 1\right)--(31) .
$$

First consider the case where $m_{1}=\alpha$. From remark 1 in section 2.2 .1 we know that in any symmetric equilibrium $b_{1} \leq m_{1}$. Since $m_{1}=\alpha$ we have $b_{1} \leq \alpha$. And for all $b_{1} \in[0, \alpha]$ we have $G\left(b_{1}\right)=0$ and $u\left(m_{1}-b_{1}, 1\right)-u\left(m_{1}, 0\right)>0$. Therefore the best possible bid when $m_{1}=\alpha$ is $b_{1}=\alpha$. That is, $b^{I}(\alpha)=\alpha$.

Now consider the case where $m_{1}>\alpha$. If bidder 1 chooses $b_{1}=\alpha$, then $\frac{\partial \pi_{1}(.)}{\partial b_{1}}>0$. Hence for this case $b_{1}>\alpha$. From (31) we can write $\frac{\partial \pi_{1}(.)}{\partial b_{1}}$ as follows (note that $G\left(b_{1}\right)>0$ for $b_{1}>\alpha$ ). 


$$
\begin{aligned}
\frac{\partial \pi_{1}(.)}{\partial b_{1}} & =G\left(b_{1}\right)\left[\frac{g\left(b_{1}\right)}{G\left(b_{1}\right)}\left[u\left(m_{1}-b_{1}, 1\right)-u\left(m_{1}, 0\right)\right]-u^{\prime}\left(m_{1}-b_{1}, 1\right)\right] \\
& =G\left(b_{1}\right)\left[(n-1) \frac{f\left(b_{1}\right)}{F\left(b_{1}\right)}\left[u\left(m_{1}-b_{1}, 1\right)-u\left(m_{1}, 0\right)\right]-u^{\prime}\left(m_{1}-b_{1}, 1\right)\right]--(32) .
\end{aligned}
$$

The above follows because

$$
\frac{g\left(b_{1}\right)}{G\left(b_{1}\right)}=(n-1) \frac{f\left(b_{1}\right)}{F\left(b_{1}\right)}
$$

Since $b_{1} \leq m_{1}$ and since the reverse hazard rate $\frac{f\left(b_{1}\right)}{F\left(b_{1}\right)}$ is non-increasing we have

$$
(n-1) \frac{f\left(b_{1}\right)}{F\left(b_{1}\right)} \geq(n-1) \frac{f\left(m_{1}\right)}{F\left(m_{1}\right)} .
$$

Since $u($.$) is strictly increasing in x$ we have for all $b_{1}<m_{1}, u\left(m_{1}-b_{1}, 1\right)>u(0,1)$ and since $m_{1} \in(\alpha, \beta]$ we have $u\left(m_{1}, 0\right) \leq u(\beta, 0)$. This implies

$$
\begin{aligned}
& (n-1) \frac{f\left(b_{1}\right)}{F\left(b_{1}\right)}\left[u\left(m_{1}-b_{1}, 1\right)-u\left(m_{1}, 0\right)\right] \\
> & (n-1) \frac{f\left(m_{1}\right)}{F\left(m_{1}\right)}[u(0,1)-u(\beta, 0)] \\
\geq & (n-1) f(\beta)[u(0,1)-u(\beta, 0)] \\
\geq & u^{\prime}(x, 1) \text { for all } x \in[0, \beta] \text { (as per the hypothesis of the proposition). }
\end{aligned}
$$

Using the above in $(32)$ we get that when $m_{1} \in(\alpha, \beta], \frac{\partial \pi_{1}(.)}{\partial b_{1}}>0$ for all $b_{1}<m_{1}$. Therefore the best bid is $b_{1}=m_{1}$. That is, $b^{I}\left(m_{1}\right)=m_{1}$.

Proof of Proposition 6 Let bidders 2,3..n bid $m_{2}, m_{3} \ldots m_{n}$. Bidder 1's income is $m_{1}$ and he chooses a bid equal to $b_{1}$. Then bidder 1 's expected payoff is

$$
\begin{aligned}
\pi_{1}\left(b_{1}, m_{1}\right) & =G\left(b_{1}\right) u\left(m_{1}-b_{1}, 1\right)+\left(1-G\left(b_{1}\right)\right) u\left(m_{1}-b_{1}, 0\right) \\
& =F^{n-1}\left(b_{1}\right) u\left(m_{1}-b_{1}, 1\right)+\left(1-F^{n-1}\left(b_{1}\right)\right) u\left(m_{1}-b_{1}, 0\right) .
\end{aligned}
$$

If bidder 1 chooses $b_{1}=m_{1}$ his expected payoff is $F^{n-1}\left(b_{1}\right) u(0,1)$. If he chooses not to bid then he gets $u\left(m_{1}, 0\right)$. If he chooses a bid strictly less than $m_{1}$ he chooses $b_{1}=m_{1}-\varepsilon$ where $\varepsilon \in\left(0, m_{1}\right)$. Then his expected payoff is

$$
F^{n-1}\left(m_{1}-\varepsilon\right) u(\varepsilon, 1)+\left(1-F^{n-1}\left(m_{1}-\varepsilon\right)\right) u(\varepsilon, 0) .
$$


Since $\forall m_{i} \in[0, \beta]$ we have (by the hypotheses of the proposition)

$$
F^{n-1}\left(m_{i}\right) u(0,1) \geq u\left(m_{i}, 0\right)
$$

and $F^{n-1}\left(m_{i}\right) u(0,1) \geq F^{n-1}\left(m_{i}-\varepsilon\right) u(\varepsilon, 1)+\left(1-F^{n-1}\left(m_{i}-\varepsilon\right)\right) u(\varepsilon, 0), \forall \varepsilon \in\left(0, m_{i}\right)$

choosing $b_{1}=m_{1}$ is optimal.

Proof of Proposition 7 The expected revenues in this case are as follows.

$$
\begin{aligned}
\text { First-price : } & R^{I}=\int_{0}^{\beta} z f_{1}(z) d z=\int_{0}^{\beta} z d F_{1}(z) \\
\text { Second-price : } & R^{I I}=\int_{0}^{\beta} z f_{2}(z) d z=\int_{0}^{\beta} z d F_{2}(z) \\
\text { All-Pay : } & R^{A P}=n \int_{0}^{\beta} z f(z) d z
\end{aligned}
$$

First note that $F_{1}(z)=F^{n}(z)$ and $F_{2}(z)=F^{n}(z)+n F^{n-1}(z)(1-F(z))$. Since $F_{1}(z)<F_{2}(z)$ for all $z \in(\alpha, \beta)$ we have $R^{I}>R^{I I}$.

Also note that

$$
\begin{aligned}
R^{I} & =\int_{0}^{\beta} z f_{1}(z) d z=n \int_{0}^{\beta} z F^{n-1}(z) f(z) d z \\
& <n \int_{0}^{\beta} z f(z) d z\left(\text { since } F^{n-1}(z)<1 \text { for all } z \in[0, \beta)\right) \\
& =R^{A P}
\end{aligned}
$$

Hence we have $R^{A P}>R^{I}>R^{I I}$. 